Portland State University

PDXScholar

7-20-1993

\title{
A Retarding-potential Analyzer for Measuring Energy Distributions in Electron Beams
}

Li Zhou

Portland State University

Follow this and additional works at: https://pdxscholar.library.pdx.edu/open_access_etds

Part of the Physics Commons

Let us know how access to this document benefits you.

\section{Recommended Citation}

Zhou, Li, "A Retarding-potential Analyzer for Measuring Energy Distributions in Electron Beams" (1993). Dissertations and Theses. Paper 4744.

https://doi.org/10.15760/etd.6628

This Thesis is brought to you for free and open access. It has been accepted for inclusion in Dissertations and Theses by an authorized administrator of PDXScholar. Please contact us if we can make this document more accessible: pdxscholar@pdx.edu. 
AN ABSTRACT OF THE THESIS OF Li Zhou for the Master of Science in Physics presented July 20,1993.

Title: A Retarding-Potential Analyzer for Measuring Energy Distributions in Electron Beams.

APPROVED BY THE MEMBERS OF THE THESIS COMMITTEE:

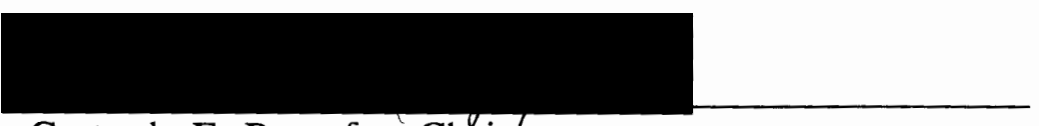

Gertrude F. Rempfer, Chair
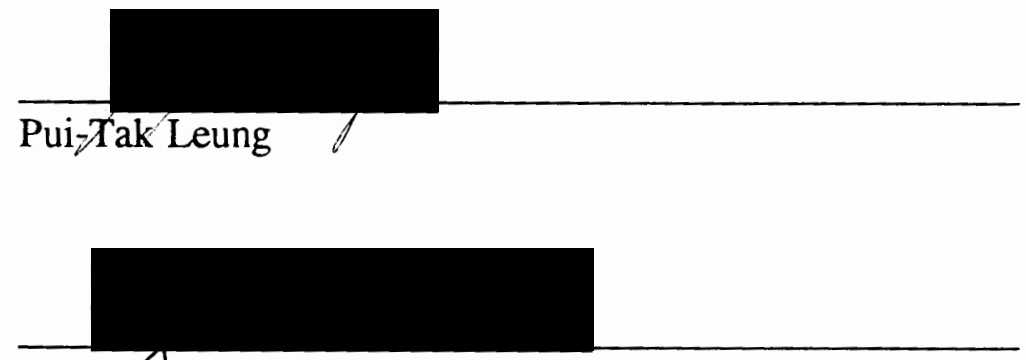

Leonard'Swanson

An energy analyzer for electron beams, based on a retarding-potential met:-od, has been studied both experimentally and theoretically. In this method a potential energy barrier at right-angles to the beam acts as a high-pass energy filter allowing electrons having energies greater than the barrier height to pass through while turning back electrons of lower energy. The potential barrier in the present study was created by applying a negative potential to an electrode consisting of a $600 /$ inch hexagonal copper 
mesh or an electrode having a pair of single apertures in series (compound aperture). Two different compound apertures, of diameters $0.4 \mathrm{~mm}$ and $1.0 \mathrm{~mm}$, were used. The retarding electrode was mounted between two grounded outer electrodes having openings centered on the axis for entrance and exit of electrons. The barrier height was adjusted by means of a small variable bias voltage between the electron gun cathode and the retarding electrode. Auxiliary electron lenses external to the retarding module were used to satisfy the conditions that the beam be normal to the retarding electrode and, for an imaging filter, that an image plane be focused at the retarding electrode.

A beam having a narrow distribution of energies was used to calibrate the energy analyzer as a function of bias voltage for the three different configurations of retarding electrode. The calibration curves were then compared with the transmission curve for a beam having a broadened energy distribution. The feasibility of obtaining a filtered image was explored by observing the image of a fine mesh focused into an aperture of the retarding electrode.

The experiments were carried out for a beam voltage of $15 \mathrm{kV}$. At this beam voltage the energy resolution is poor, being 3.6 volts for the smaller compound aperture, 3.7 volts for the mesh, and 5.2 volts for the larger compound aperture. Typically, electrostatic analyzers operate on a beam which has been decelerated to low energies before entering the analyzer, in which case the energy resolution is proportionately better. For example, at $150 \mathrm{~V}$ the above resolution figures would be $0.036,0.037$, and 0.052 , respectively. In view of the potential for high energy-resolution and the attractive features of in-line, rotationally-symmetric systems, it is felt that the retarding-potential analyzer is a promising candidate for further development. 
A RETARDING-POTENTIAL ANALYZER FOR MEASURING

ENERGY DISTRIBUTIONS IN ELECTRON BEAMS

by

LI ZHOU

A thesis submitted in partial fulfillment of the requirements for the degree of

\section{MASTER OF SCIENCE \\ in \\ PHYSICS}

Portland State University

1993 


\section{TO THE OFFICE OF GRADUATE STUDIES:}

The members of the Committee approve the thesis of Li Zhou presented July 20, 1993.

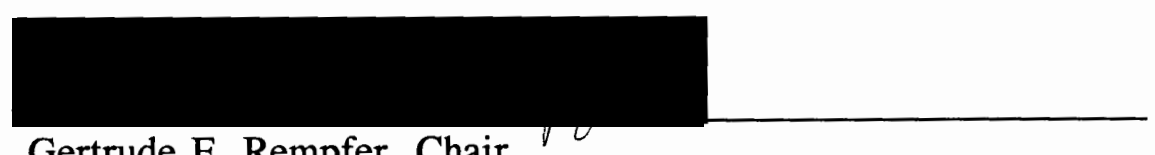

Gertrude F. Rempfer, Chair
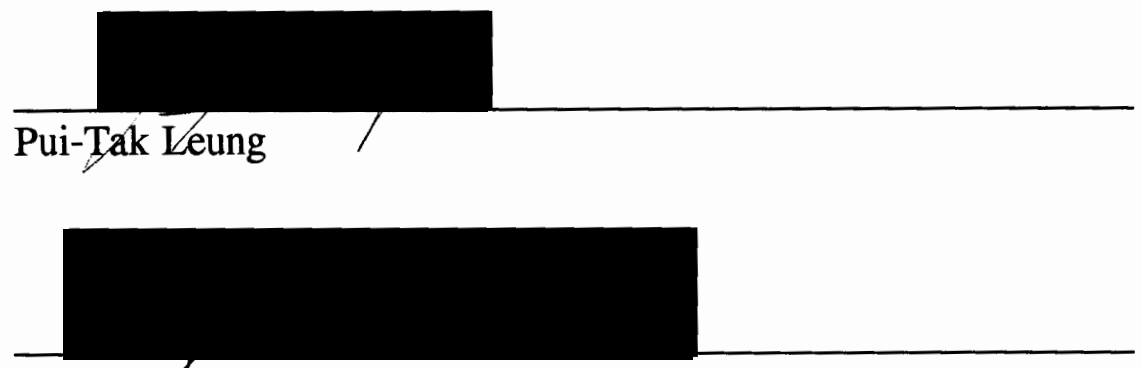

Leonard \$wanson

\section{APPROVED:}

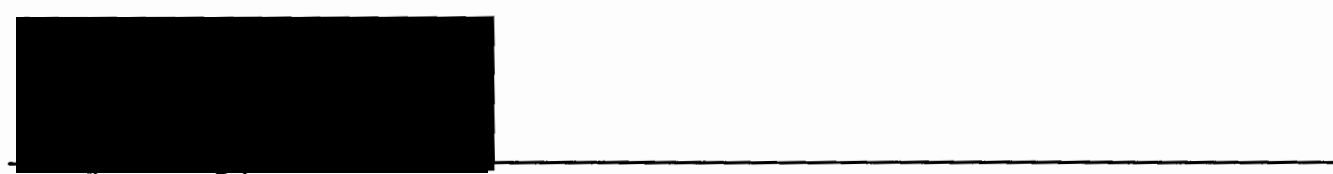

Eric Bodegom, Chair, Department of Physics

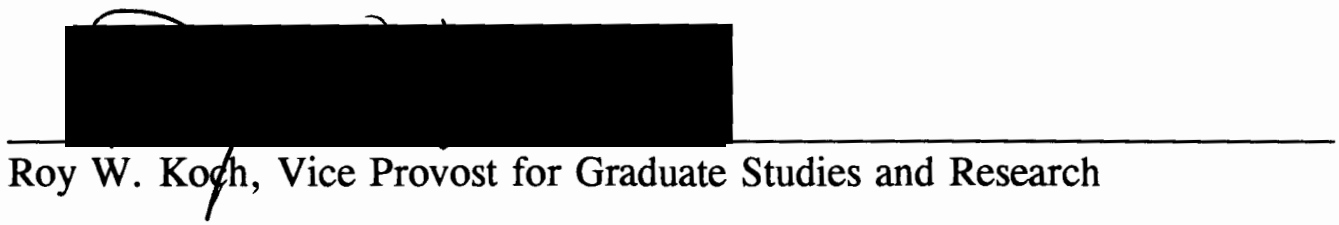




\section{ACKNOWLEDGMENTS}

I would like to thank the following people for their gracious assistance with this thesis:

Members of my thesis committee, Gertrude F. Rempfer, Pui-Tak Leung, Leonard Swanson.

Especially Professor Gertrude F. Rempfer for spending much time introducing me to the electron optics experiment, and for reading and discussing my thesis paper.

Mr. Walt Skoczylas and Mr. Denis Desloge for help in my experiment.

I am grateful also for assistance from the M.J. Murdock Charitable Trust. 
TABLE OF CONTENTS

PAGE

ACKNOWLEDGEMENTS $\ldots \ldots \ldots \ldots \ldots \ldots \ldots$ iii

LIST OF FIGURES $\ldots \ldots \ldots \ldots \ldots \ldots \ldots \ldots \ldots \ldots \ldots$

\section{CHAPTER}

I INTRODUCTION. $\ldots \ldots \ldots \ldots \ldots \ldots \ldots \ldots \ldots$

II THE RETARDING-POTENTIAL METHOD $\ldots \ldots \ldots \ldots \ldots$

The Retarding-Potential Configuration . . . . . . . 9

Equivalent Optical System of the Retarding-Potential Analyzer 12

The Auxiliary Lens System $\ldots \ldots \ldots$. . . . . 17

The Output Optics .............. . . 20

III EXPERIMENTAL PROCEDURE $\ldots \ldots \ldots \ldots \ldots \ldots . \ldots . \ldots$

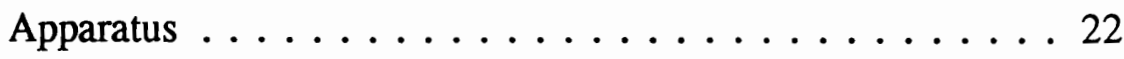

Electron-Optical Settings . . . . . . . . . . . 25

Calibration of the Analyzer $\ldots \ldots \ldots \ldots 28$

Energy-Distribution Measurement . . . . . . . . . . 29

Imaging a Filtered Beam $\ldots \ldots \ldots \ldots$. . . . 30

IV EXPERIMENTAL RESULTS AND DISCUSSION $\ldots \ldots \ldots 31$

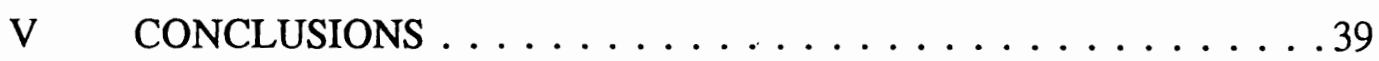




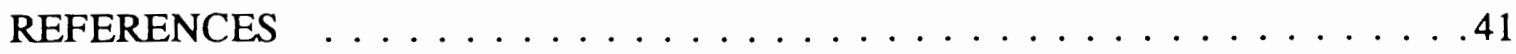

APPENDICES

A POTENTIAL DISTRIBUTION DUE TO A CIRCULAR HOLE IN AN ELECTRODE SEPARATING TWO UNIFORM FIELDS . . 42

B THE DAVISSON-CALBICK FORMULA FOR THE FOCAL

LENGTH OF AN APERTURE LENS . . . . . . . . 46 


\section{LIST OF FIGURES}

FIGURE

PAGE

1. Cross Section of a Cylindrical Mirror Analyzer . . . . . . . . 2

2. Schematic Axial Section of a $90^{\circ}$ Spherical Deflector Analyzer . . . 4

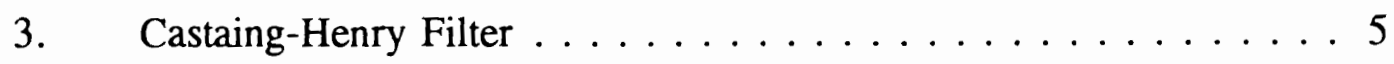

4. Omega Filter $\ldots \ldots \ldots \ldots \ldots \ldots$

5. Schematic Diagram of the Retarding-Potential Analyzer ....... 10

6. Schematic Diagram of the Analyzer with a Compound Aperture . . 11

7. Schematic Diagram of the Input Side of the Retarding-Potential Energy Analyzer ..................... 14

8. Equivalent Optical System of the Input Side of the Energy

Analyzer (Shown in Figure 7$) \ldots \ldots \ldots$

9. Electron-Optics of the Input Side of the Analyzer . . . . . . . . 19

10. Electron-Optics of the Output Side of the Analyzer . . . . . . . 21

11. Essential Dimensions of the Compound Apertures Used

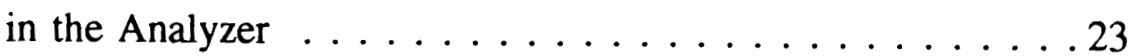

12. Photograph of Experimental System Setup . . . . . . . . . . . 24

13. Schematic Diagram of the Experimental System ......... 26

14. Essential Dimensions of Experimental System _... . . . . . 27 
15. Calibration Curve for $1 / 600 "$ Hexagonal Mesh .......... . . 32

16. Calibration Curve for $0.4 \mathrm{~mm}$ Compound Aperture ........ 33

17. Calibration Curve for $1.0 \mathrm{~mm}$ Compound Aperture . . . . . . . 34

18. Transmission Curve for an Energy-Broadened Beam . . . . . . . 36

19. Potential Distribution about a Circular Aperture . . . . . . . . . . 44

20. Circular Aperture in an Electrode with an Axial Electric Field on One Side of the Electrode . . . . . . . . . . . . 47

21. Diverging Action Around the Aperture .............. 49

22. Cylindrical Volume Diagram for Calculating the Integral . . . . . 50 


\section{CHAPTER I}

\section{INTRODUCTION}

An electron energy analyzer is a device which separates electrons having different energies. Analyzers are used to measure the distribution of energies, for example, in a beam emitted from an electron source or after interaction with a specimen. Analyzers are also used to separate out a portion of a beam with a narrow distribution of energies, for illuminating a specimen or for other purposes. One of the principal uses for electron energy analyzers is in electron spectroscopy for chemical analysis (ESCA) [1], [2]. In this technique the energy spectrum of electrons emitted from a specimen under the influence of $\mathrm{x}$ rays is used to determine the chemical nature of the specimen. Energy analyzers are also used in electron diffraction studies, and as attachments to electron microscopes for spectroscopic imaging of specimens [3].

There are a number of different types of energy analyzers. Most are based on separation by deflection in electrostatic or magnetic fields. The most commonly used electrostatic analyzers are the cylindrical mirror analyzer (CMA), the cylindrical analyzer (CA), and the spherical deflector analyzer (SDA).

The CMA is shown in figure 1. It has two co-axial cylinders, one within the other, between which a potential difference is applied. There are two longitudinal slits on the inner cylinder. The slits are separated in the axial direction. Electrons from a 


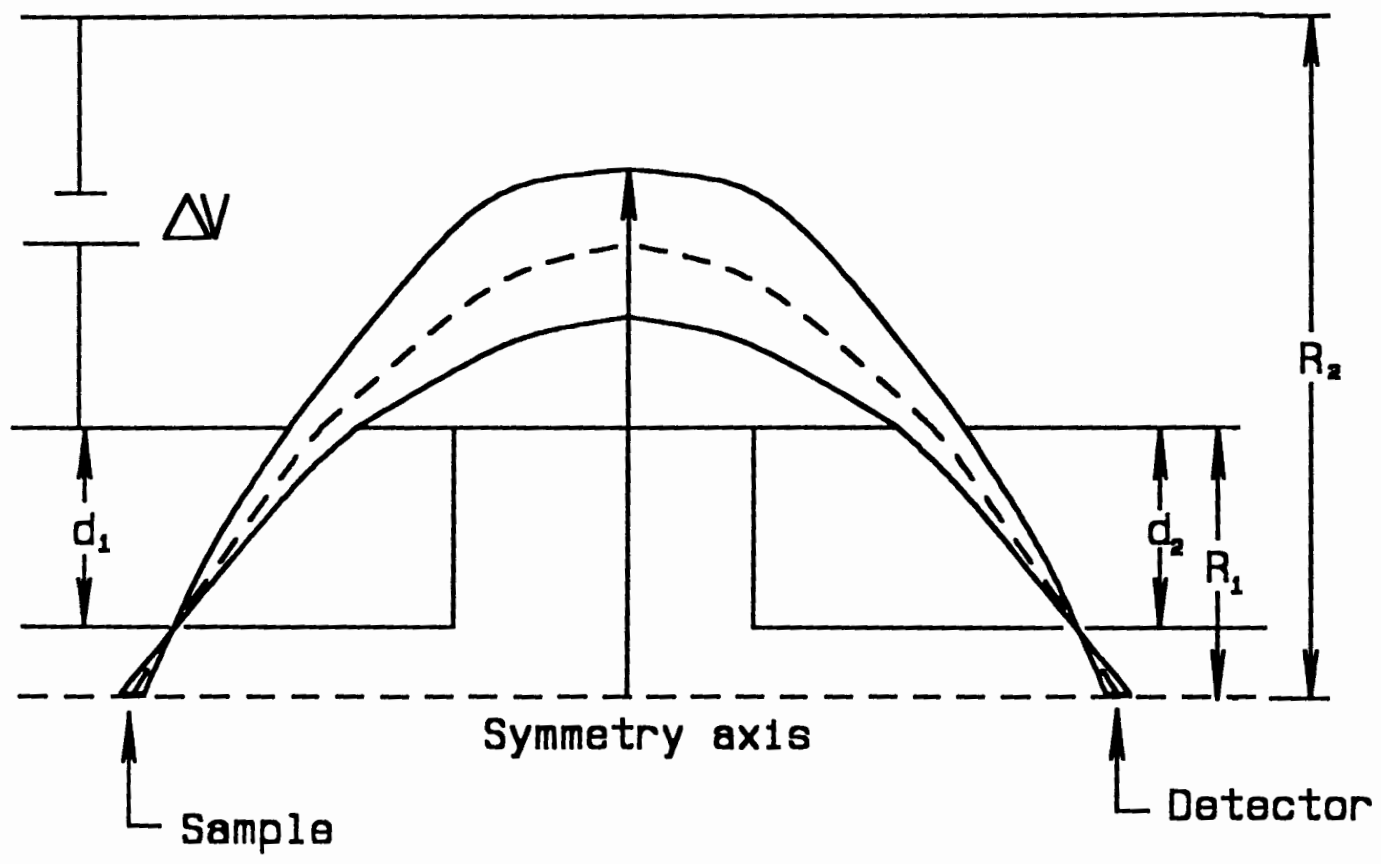

Figure 1. Cross section of a cylindrical mirror analyzer. Selected trajectories are shown. 
specimen on the axis enter the space between the cylinders through the first slit. The cylindrical electric field acts as a mirror, reflecting and focusing the electrons. The potential difference between the cylinders determines the energies of the electrons which are focused into the second slit and recorded by the detector. The CMA is very compact and offers good energy resolution.

In the $\mathrm{CA}$ the electrons also travel in the electric field between two coaxial cylinders (or sectors thereof), but in this case the electron orbits are in a plane normal to the axis, rather than parallel to it as in the CMA. The entrance and exit slits are parallel to the axis and are separated $137^{\circ}$ in azimuth. Electrons passing through the entrance slit refocus in the plane of the exit slit, but at different distances from the axis depending on their energies. The potential difference between the cylinders determines the energies at which the electrons are focused into the exit slit.

The spherical sector analyzer has two concentric spherical electrodes, between which a potential difference is applied, creating an inverse-square field in which the electrons travel. Usually the electrodes are hemispherical, or 1/4 spherical as shown in figure 2. The SDA offers the best compromise between beam current and energy resolution.

Energy analyzers based on magnetic deflection include the Castaing-Henry energy filter and the Omega filter. The Castaing-Henry analyzer consists of a magnetic prism and an electrostatic mirror, as shown in figure 3. Electrons entering the analyzer are first deflected by the magnetic prism through $90^{\circ}$ onto the mirror axis. They are reflected by the mirror so that they pass a second time through the magnetic prism. Since the electron 


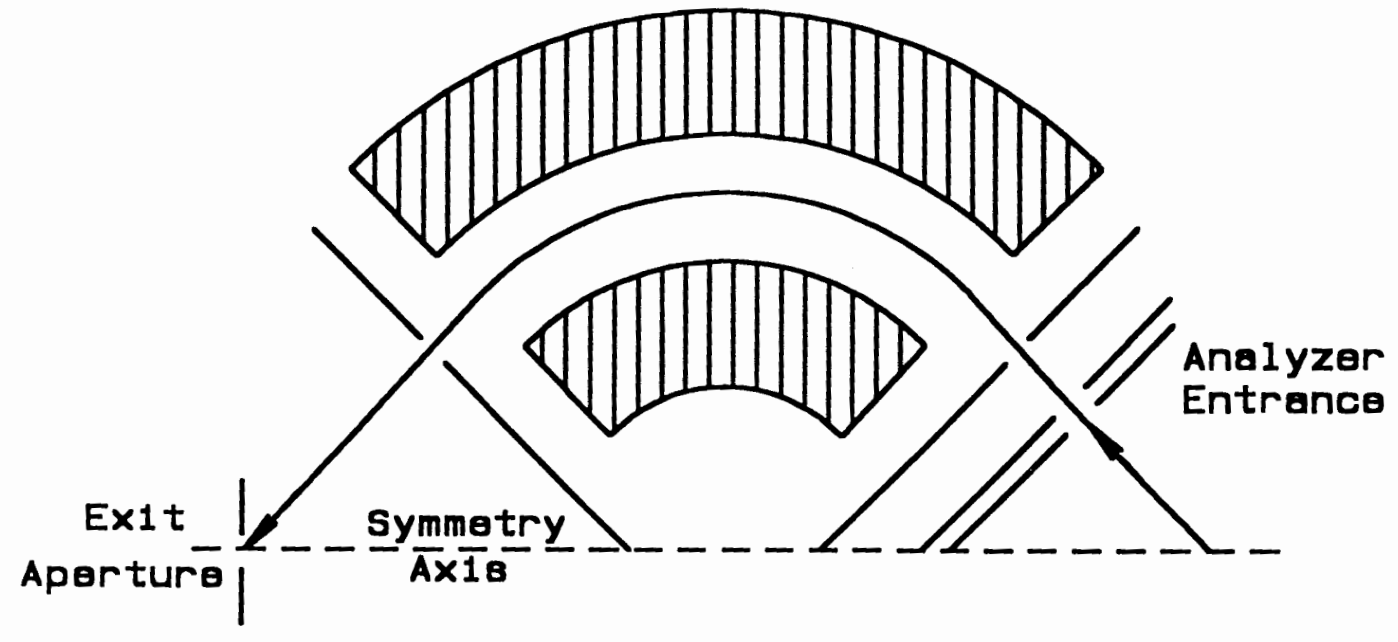

Figure 2. Schematic axial section of a $90^{\circ}$ spherical deflector analyzer. 


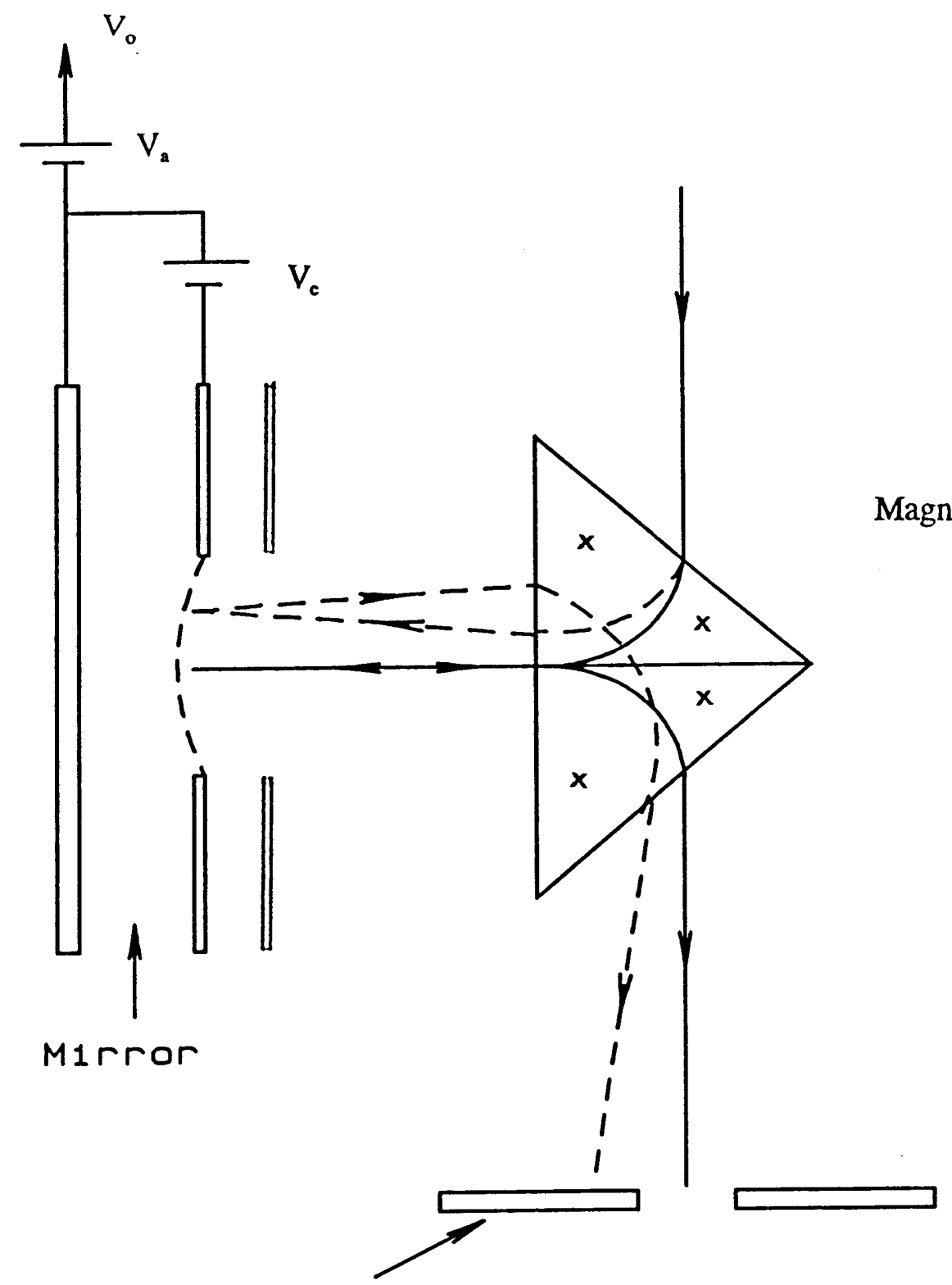

Energy-Selecting Slit

Figure 3. Castaing-Henry filter. Trajectories of the no-loss (solid line) and energy-loss (dashed line) electrons are shown. 
velocity is now reversed, the second magnetic deflection restores the beam to its original direction. The Castaing-Henry filter is used in electron microscopes for spectroscopic imaging. The analyzer can be set to transmit no-loss electrons (solid line), as shown in figure 3, or electrons with a specified energy-loss (dashed line). The rays in the energy selecting plane appear to come from an achromatic virtual image which is then focused into a selected-energy real image by the projection lenses.

The Omega filter is shown in figure 4 . The electrons pass through a magnetic prism and are deflected through an angle, typically $90^{\circ}-120^{\circ}$. Instead of being reflected by an electrostatic mirror (as in the Castaing-Henry system) they then enter a second prism whose magnetic field is in the reverse direction, so that the beam emerges parallel to its original direction. A further two prisms are located symmetrically with respect to the first pair, and the complete trajectory takes the form of the Greek letter $\Omega$. The filtered beam emerges from the device along its original axis. Whereas the CastaingHenry filter requires a high-voltage connection to the mirror electrode, the Omega filter does not require such a connection and is therefore better suited for use with high-voltage electron microscopes.

Two methods of energy analysis which do not depend on angular dispersion are the time-of-flight method and the retarding-potential method. The energy analyzer discussed in the present thesis is based on the retarding-potential method. The operating principle of this method is that when a potential energy barrier is placed in the path of the electron beam and at right angles to the direction of the beam, electrons with energies greater than the barrier height are transmitted whereas electrons of lower energy are 


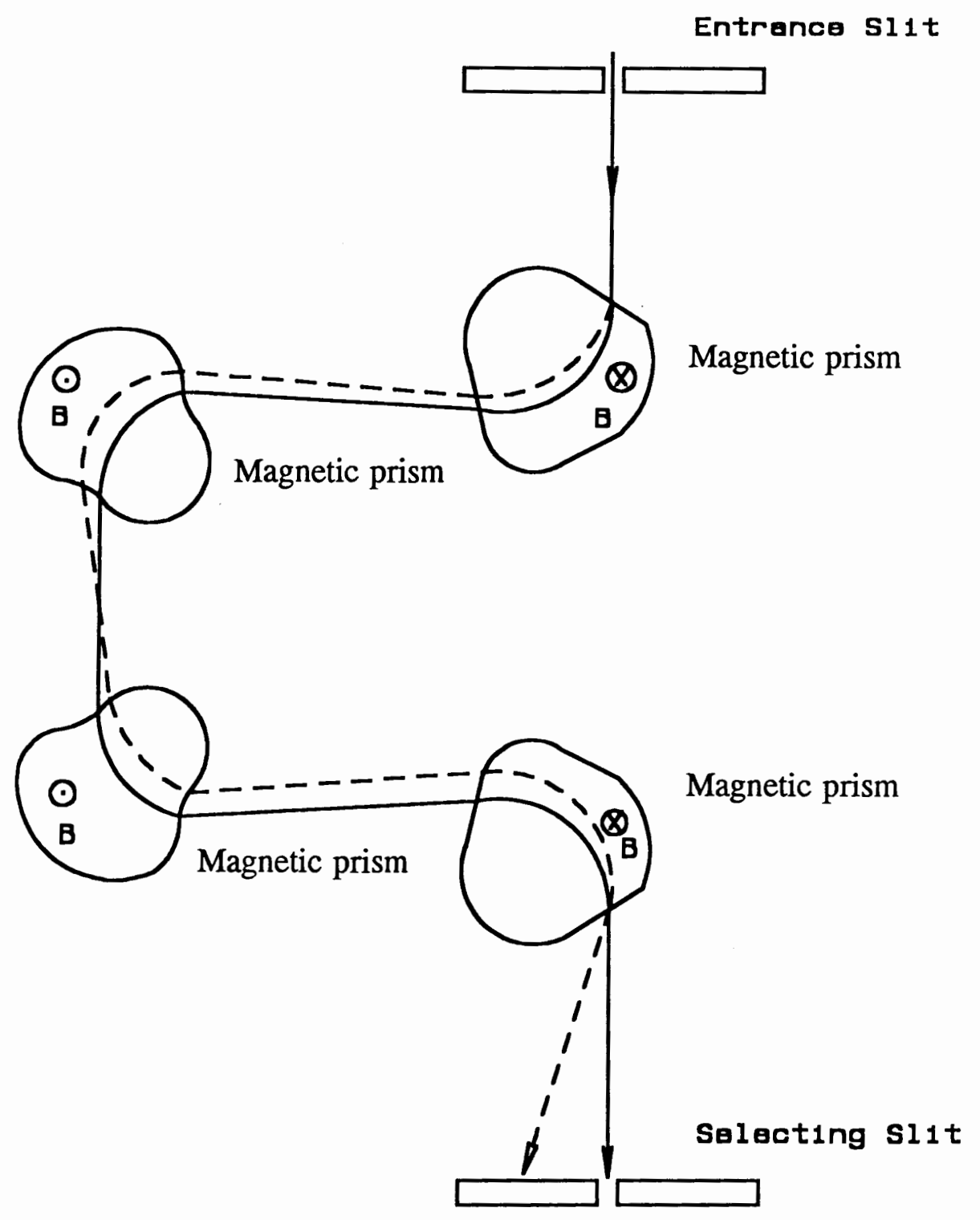

Figure 4. Omega filter. Trajectories of the no-loss (solid line) and energyloss (dashed line) electrons are shown. 
turned back. By measuring the transmitted current of electrons as a function of barrier height, one can determine the energy distribution in the beam. The present investigation builds on earlier work in this laboratory carried out by John P. Stewart for his Master's degree thesis research [4]. Stewart used the retarding-potential method to measure the energy losses of electrons scattered through small angles on passing through a thin aluminum foil. A potential barrier which allows electrons to be transmitted can be created by applying a negative potential to an electrode consisting of a fine mesh, or having a single small opening, through which electrons having sufficient energy can pass. Two such retarding electrodes can be used in series with a small gap between them for a more uniform height of barrier. The electric fields around the openings in the retarding electrode have focusing effects which scatter the beam as it passes through the openings. In general this scattering interferes with using the barrier as an imaging filter. However, if the barrier is located in an image plane, the beam can in principle be refocused to an image in spite of the scattering effect of the openings in the retarding electrode. In this study the energy-analyzing properties of the retarding-potential method are investigated with two different types of retarding electrodes, and the feasibility of forming a filtered image is explored. 


\section{CHAPTER II}

\section{THE RETARDING-POTENTIAL METHOD}

In the retarding-potential method discussed here, the retarding-potential module is used in combination with an exterior electron lens system. Because of electron optical effects in the retarding arrangement, an input lens is needed to focus the beam so that it is normal to the retarding electrode. Furthermore, if the module is to be used as an imaging filter, the input electron optics must have the capability of focusing an image of the specimen at the retarding electrode, and output optics must be included to re-focus the filtered image.

\section{THE RETARDING-POTENTIAL CONFIGURATION}

The retarding electrode is supported by insulating spacers between two outer electrodes at ground potential. The outer electrodes have openings centered on the axis for passage of electrons into and out of the analyzer. Two types of retarding electrodes, shown schematically in figures 5 and 6, were investigated. In figure 5 the retarding electrode is a fine mesh. In figure 6 the retarding electrode has a pair of single openings in series (compound aperture). The potential barrier for the electrons is created by the application of a negative potential to the retarding electrode. As shown, the retarding electrode is connected to cathode potential $\mathrm{V}_{\mathrm{C}}$ through a bias supply which provides a 
Adjugtablo Biag Voltago

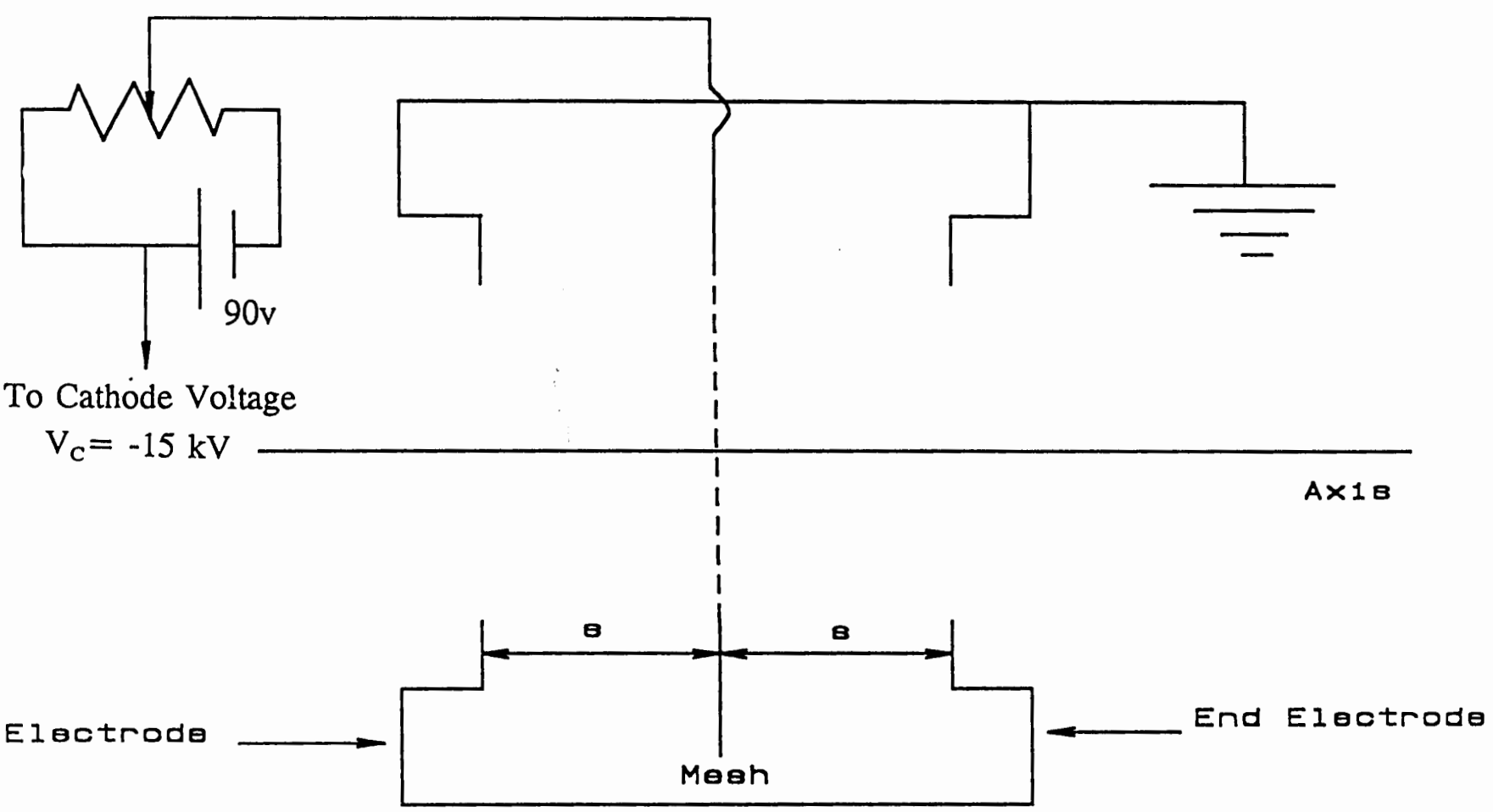

Figure 5. Schematic diagram of the retarding-potential analyzer. 
Adjustable Blas Voltage

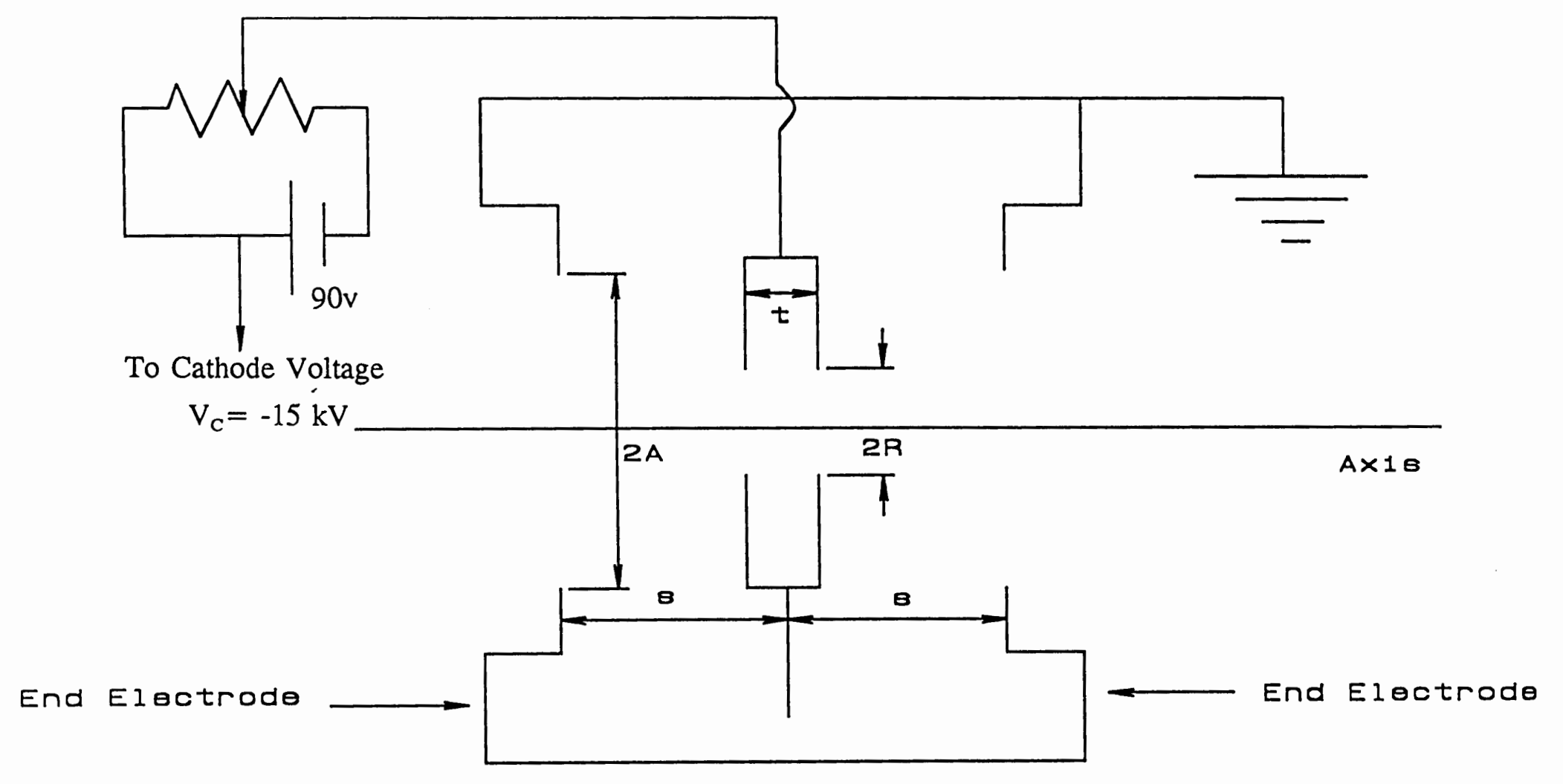

Figure 6. Schematic diagram of the analyzer with a compound aperture. 
small adjustable voltage $V_{\text {bias }}$ for changing the height of the potential barrier. The potential of the retarding electrode is $V_{M}=V_{C}+V_{\text {bias. }}$. The retarding electrode acts like a filter, allowing electrons having energies above a certain threshold, depending on the bias, to pass through while returning those of lower energy. Because of the field between the retarding electrode and the grounded end electrodes, the potential in the openings of the retarding electrode is less negative than that of the electrode. Therefore, a negative bias on the electrode, relative to cathode, is needed to cut-off transmission of the beam entirely. A theoretical calculation of the cut-off bias voltage for the retarding mesh electrode is given in Appendix A. The change in intensity of the transmitted beam as the bias is made less negative than cut-off is used to obtain the energy distribution in the beam. The energy resolution is limited by the voltage interval between cut-off and full transmission.

Electron-optical effects in the analyzer due to the electric fields between electrodes, and around the apertures in the outer electrodes, cause the effective position and size of the retarding electrode, when referred to the space outside the analyzer, to appear different from its actual size and position. These optical effects are analyzed in the following section.

\section{EQUIVALENT OPTICAL SYSTEM OF THE RETARDING-POTENTIAL ANAĹYZER}

The input and output sides of the retarding module are symmetrical, and since the analyzer is operated with the beam normal to the retarding electrode, the equivalent optical system is worked out for one side only (the input side), and is illustrated for the 
case of the mesh electrode as shown in figure 7.

The electric field between the outer electrode $A$ at potential $V_{A}\left(V_{A}=0\right.$ in the experiments) and the mesh $M_{o}$ at a negative potential $V_{M}$ can be thought of as a combination of two separate fields: (i) a non-uniform field in the immediate vicinity of the aperture in A, followed by (ii) a uniform (retarding) field between electrodes A and $M_{\mathrm{o}}$. (For this calculation the short-range local fields around the small openings in the mesh can be ignored.)

(i) The aperture field. The field around the aperture in electrode A acts like a thin lens. This was first noted by Davisson and Calbick [5]. (See also the detailed derivation in Appendix B.) If the variation in potential in the plane of the aperture is negligible relative to the beam voltage of the electrons at the aperture, the focal length of the aperture lens is

$$
f=\frac{4 V_{B}}{(\partial V / \partial z)_{2}-(\partial V / \partial z)_{1}}
$$

$V_{B}$ is the beam voltage (defined by $\mathrm{eV}_{B}=\mathrm{mv}^{2} / 2$ ) of the electrons at the potential of the aperture electrode, and is equal to $V_{A}-V_{C}$, where $V_{C}$ is the potential of the cathode from which the electrons are emitted. $(\partial \mathrm{V} / \partial \mathrm{z})_{2}$ and $(\partial \mathrm{V} / \partial \mathrm{z})_{1}$ are the potential gradients (in the direction of electron motion) on the two faces of the electrode in the absence of the aperture. Their values are

$$
(\partial V / \partial z)_{2}=\frac{V_{M}-V_{A}}{S} ; \quad(\partial V / \partial z)_{1}=0
$$

where $\mathrm{s}$ is the separation between $\mathrm{A}$ and $\mathrm{M}_{\mathrm{o}}$. 


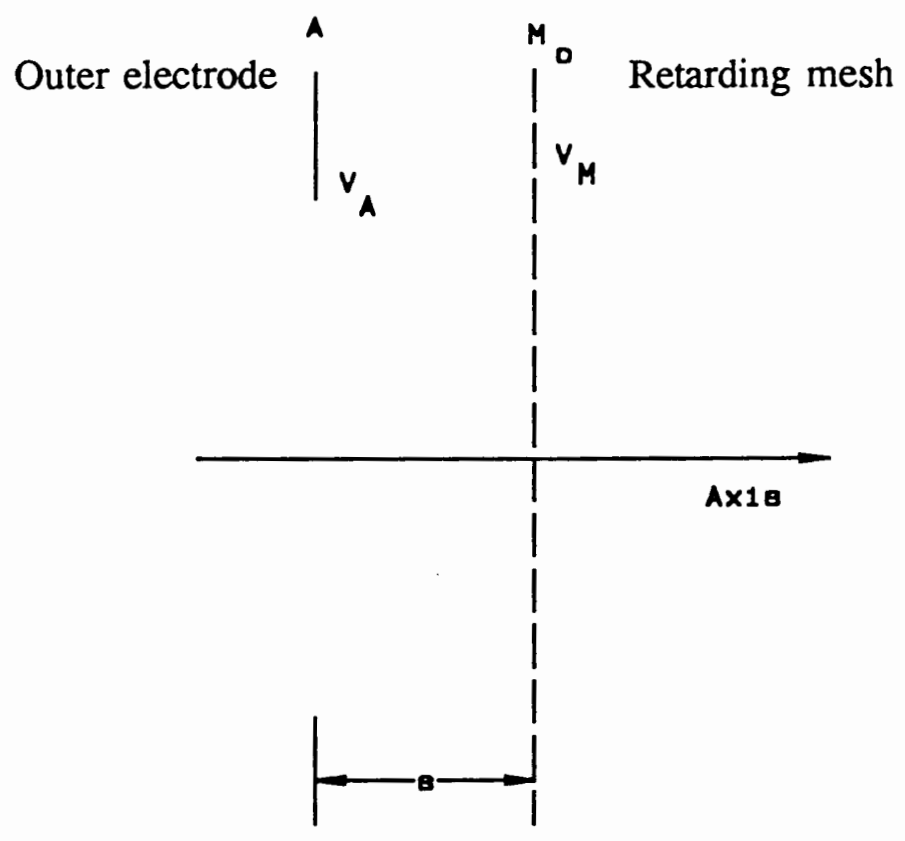

Figure 7. Schematic diagram of the input side of the retarding-potential energy analyzer. Radial distances have been exaggerated for clarity. The potential $V_{M}$ of the mesh is close to the potential $V_{C}$ of the cathode from which the electrons are emitted. The potential $V_{A}$ of the outer electrode is positive with respect to $V_{M}$. 
Substitution in equation (1) gives

$$
f_{A}=\frac{4\left(V_{A}-V_{C}\right) s}{\left(V_{M}-V_{A}\right)}=\frac{-4\left(V_{A}-V_{C}\right) s}{\left(V_{A}-V_{C}-V_{\text {bias }}\right)}
$$

Since in our experiments $V_{\text {bias }}<<V_{A}-V_{C}$, equation (2) reduces to

$$
f_{A}=-4 s
$$

Thus, the aperture field acts like a diverging lens of focal length $-4 \mathrm{~s}$.

(ii) The uniform retarding field. The effect of the uniform retarding field is to cause the electron paths to curve along parabolic arcs. As the electrons enter the retarding field, the tangents to their paths point to where the electrons would go if the potential remained equal to $\mathrm{V}_{\mathrm{A}}$. However, because the potential becomes increasingly negative, the actual distance traveled into the field is shorter than the virtual distance indicated by the tangents. In the present experiments, where the electrons are decelerated to close-to-zero velocities at the retarding electrode, the virtual distance to the electrode indicated by the initial tangents to the electron path is (to first order) twice as large as the actual distance.

The combined electron optical effects of (i) and (ii) are shown in figure 8. Figure 8 is an equivalent optical representation of the input side of the analyzer (figure 7). The aperture field is represented by a thin diverging lens of focal length $-4 \mathrm{~s}$, and the retarding mesh $M_{0}$ is replaced by the virtual mesh $M_{o}^{* *}$ referred to field-free space at potential $V_{A}$. $M_{o}^{*}$ is the same size as $M_{o}$, but is twice as far from electrode A. Curved rays in actual space are transformed into straight lines in virtual space. It is convenient to work with virtual space, not only because the rays are straight, but also because the virtual space 


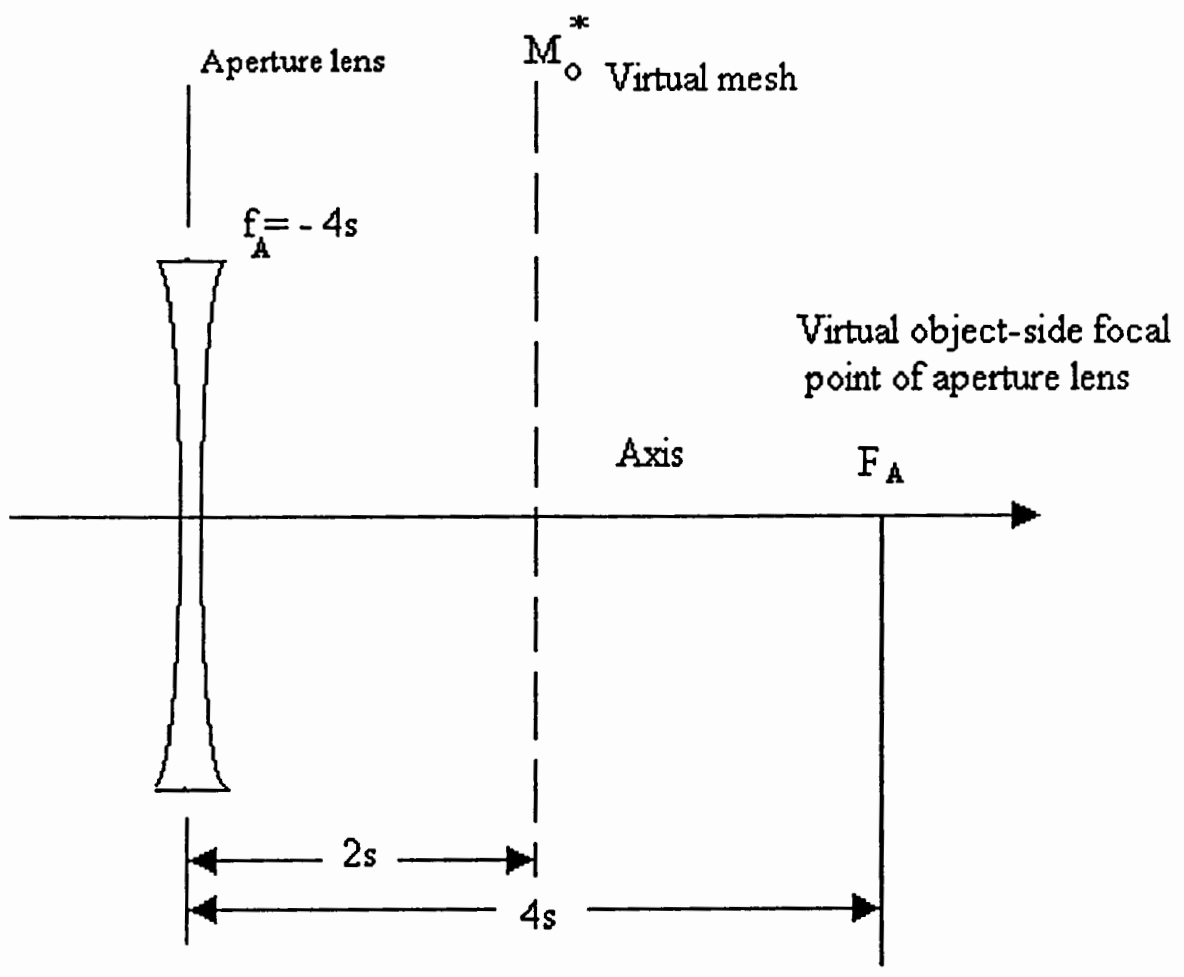

Figure 8. Equivalent optical system of the input side of the energy analyzer (shown in figure 7). The retarding field of length $s$ between $A$ and $M_{O}$ in figure 7, when referred to potential $V_{A}$ becomes a virtual space of length $2 \mathrm{~s}$ between $A$ and the virtual mesh $\mathrm{M}_{\mathrm{O}}{ }^{*}$. The electrical field around the aperture in A acts as a diverging aperture lens of focal length $\mathrm{f}_{\mathrm{A}}=-4 \mathrm{~s}$. 
inside the analyzer is the image space of the aperture lens.

The electron beam to be analyzed consists of pencils of rays coming from the individual points of a specimen or other object. The conditions on the beam inside the analyzer are (a) that the axes of the ray pencils be parallel to the optical axis, and normal to the retarding mesh; and (b) that, if one seeks to obtain an filtered image of the specimen, the rays in the individual pencils should focus to an intermediate image at the mesh.

With regard to condition (a), since the ray axes inside the analyzer are parallel in the image space of the aperture lens, the ray axes outside the analyzer must be directed toward the virtual focal point of the aperture lens, a distance $4 s$ beyond the lens.

Condition (b) requires that the rays forming the individual pencils focus at the mesh $\mathbf{M}_{0}$, or equivalently that the virtual rays focus at the virtual mesh $\mathbf{M}_{0}{ }^{*}$ in the image space of the aperture lens. The image distance is thus $\mathrm{AM}_{\mathrm{o}}{ }^{*}=2 \mathrm{~s}$. The object distance AM toward which the pencil rays should be directed from outside the analyzer is given by the thin-lens formula

$$
1 / \mathrm{AM}+1 / \mathrm{AM}_{\mathrm{o}}^{*}=1 / \mathrm{f}_{\mathrm{A}} .
$$

Substituting $\mathrm{f}_{\mathrm{A}}=-4 \mathrm{~s}$ and $\mathrm{AM}_{\mathrm{o}}{ }^{*}=2 \mathrm{~s}$ gives $\mathrm{AM}=-4 / 3 \mathrm{~s}$, which means that the object is virtual, and at a distance $4 / 3 \mathrm{~s}$ beyond the aperture lens. The virtual object is $2 / 3$ the size of the virtual mesh $\mathbf{M}_{0}^{*}$.

\section{THE AUXILIARY LENS SYSTEM}

Prefocusing the beam as prescribed in the foregoing section can be accomplished 
by means of auxiliary lenses external to the analyzer. See figure 9 , where the optics is illustrated for a single off-axis ray pencil. The input lens is a converging lens which focuses the ray axes toward a point $4 \mathrm{~s}$ beyond the aperture lens. At the same time, if an image of the specimen is to occur at the retarding mesh, the rays of each pencil are focused to form a virtual image of the specimen at $\mathrm{M}$, a distance $4 / 3 \mathrm{~s}$ beyond the aperture.

The ray-axis focus at $4 \mathrm{~s}$ and the virtual image at $4 / 3 \mathrm{~s}$ beyond the aperture lens are the images for the input lens. If the input lens is spaced a distance $d_{i}$ from the aperture lens, the image distance $z_{a}{ }^{\prime}$ to the ray-axis focus is $d_{i}+4 s$. The conjugate distance $z_{\mathbf{a}}$ where the ray pencils cross the axis on the object side of the input lens is given by

$$
1 / z_{\mathrm{a}}+1 /\left(\mathrm{d}_{\mathrm{i}}+4 \mathrm{~s}\right)=1 / \mathrm{f}_{\mathrm{i}}
$$

where $f_{i}$ is the focal length of the input lens. Likewise, the image distance $z_{b}{ }^{\prime}$ for the image plane at $M$ is $d_{i}+4 / 3 \mathrm{~s}$, and the conjugate object distance $z_{b}$ is given by

$$
1 / z_{b}+1 /\left(d_{i}+4 / 3 s\right)=1 / f_{i} .
$$

From equations (5) and (6) it can be seen that $z_{b}$ is larger than $z_{a}$. Hence the ray pencils must cross the axis between $z_{b}$ and the input lens, and at $z_{b}$ must be converging toward the axis. The purpose of the field lens in figure 9 is to direct the ray pencils so that they cross the axis at $z_{\mathbf{a}}$. Either an image of the specimen, or the specimen itself, can be located at $\mathrm{z}_{\mathrm{b}}$. The field lens can be placed in an image plane, or before or immediately after a specimen. 


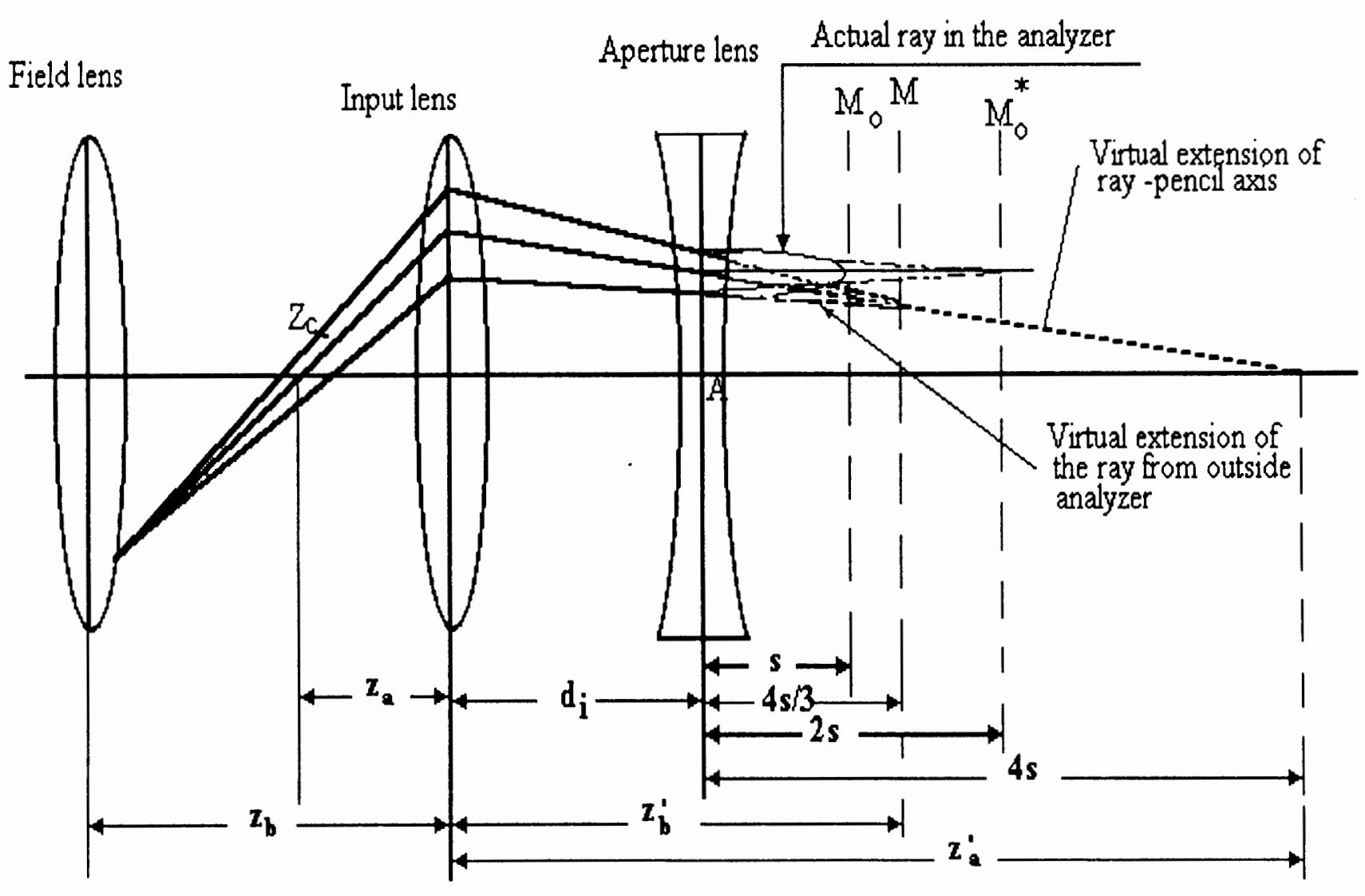

Figure 9. Electron-optics of the input side of the analyzer. 


\section{THE OUTPUT OPTICS}

The equivalent optical system for the output side of the analyzer is the mirror image of the optics for the input side. The virtual space between the mesh and the outer electrode on the output side is now the object space for the aperture lens, and the distance $2 s$ to the virtual mesh $M_{0}^{*}$ is the object distance. A virtual image $M$ of $M_{0}^{*}$ is formed at a distance of $4 / 3 \mathrm{~s}$ upstream from the aperture. It is $2 / 3$ the size of $M_{o}^{*}$ (and $\mathbf{M}_{\mathrm{o}}$ ), and hence is the same size as the virtual image $\mathrm{M}$ projected into the analyzer by the input lens in figure 9. The output lens can be used to collimate the filtered beam or, as illustrated in figure 10 , to form a filtered image.

The changes in transmitted beam current resulting from incremental changes in the barrier height give information as to the energy distribution in the beam, and changes in contrast in the filtered image give further information as to the spacial distribution of the energies. For good energy resolution the voltage interval over which the transmission rises from cut-off to substantially full transmission (the rise interval) should be small for a given energy. A small rise interval can be achieved by decelerating the beam to low voltages before it enters the analyzer. This is because the potential difference between the end electrode and the retarding electrode can be correspondingly smaller, and the variation in potential in the plane of the aperture is smaller. In the present experiments the beam was not decelerated since the principal interest was in studying the transmission characteristics of the analyzer. In fact, the large rise-interval was useful in that it enabled the beam from the electron gun, with a small energy spread by comparison, to be used for calibrating the analyzer. 


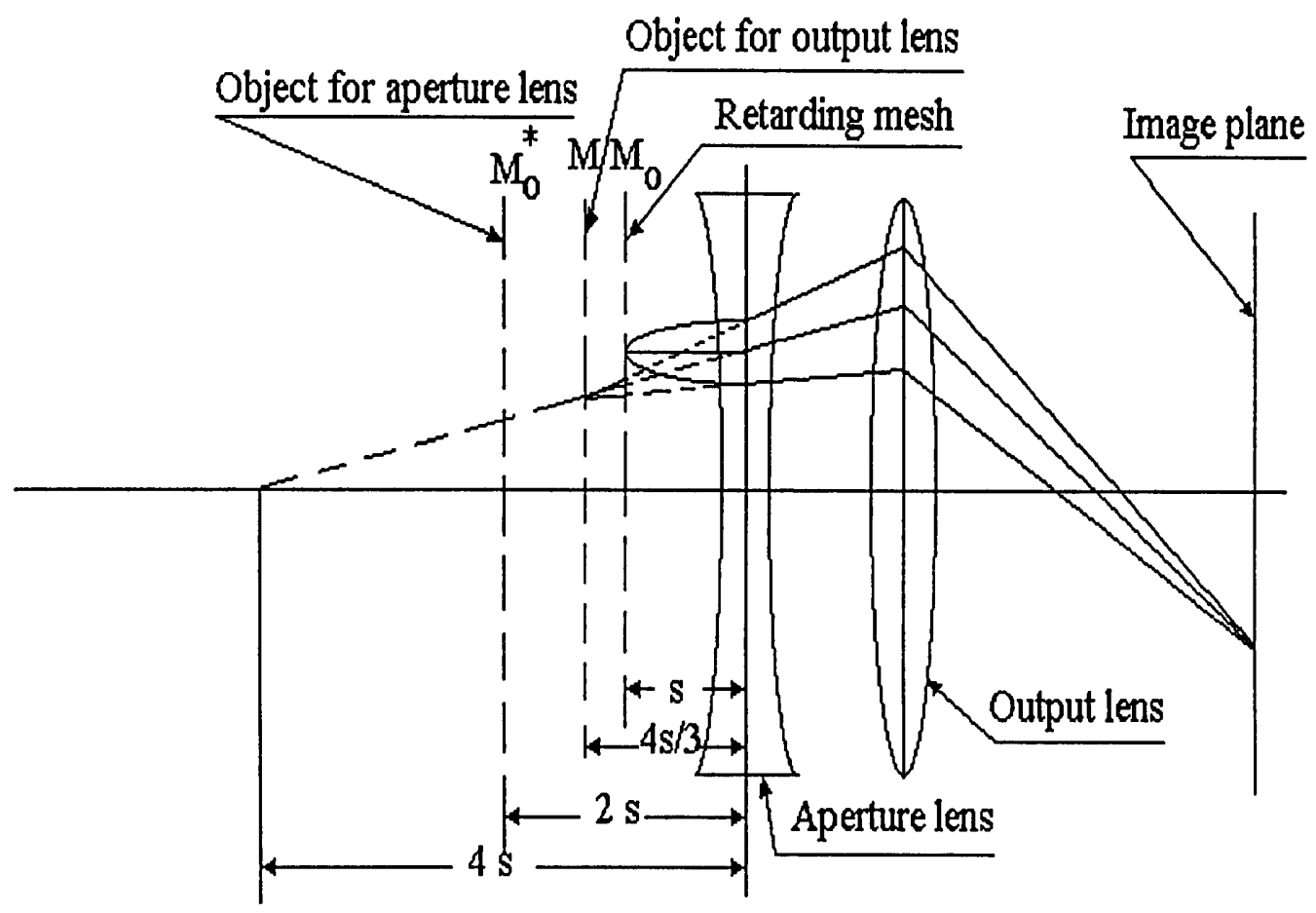

Figure 10. Electron-optics of the output side of the analyzer. 


\section{CHAPTER III}

\section{EXPERIMENTAL PROCEDURE}

Experiments were carried through with a hexagonal mesh of pitch 600/inch and with electrodes having compound apertures of two different diameters, $1.0 \mathrm{~mm}$ and 0.4 $\mathrm{mm}$, serving as the retarding electrode. The geometry of the compound apertures is given in figure 11. The discussion of the experiments is organized into the following topics: apparatus, electron-optical settings, calibration of the analyzer, energy-loss measurement, and the filtered image.

\section{APPARATUS}

The experiments were conducted in a horizontal electron-optical vacuum bench at pressures $\leq 10^{-4}$ Torr. A photograph of the bench is shown in figure 12 . The bench is fitted with a V-way for supporting and aligning the electron optical components. A thermionic electron gun (1) is mounted at the left end of the bench, and a phosphorcoated fiber optics window or camera (9) is mounted at the other end. The electron beam from the gun is focused by a condenser lens (2) into a pinhole, which serves as the electron source for the experiment. The beam from the pinhole is then focused by the field lens (3) so that, in combination with the input lens (4), the beam is normal to the retarding electrode of the analyzer (5). A specimen, if used, is placed downstream from 


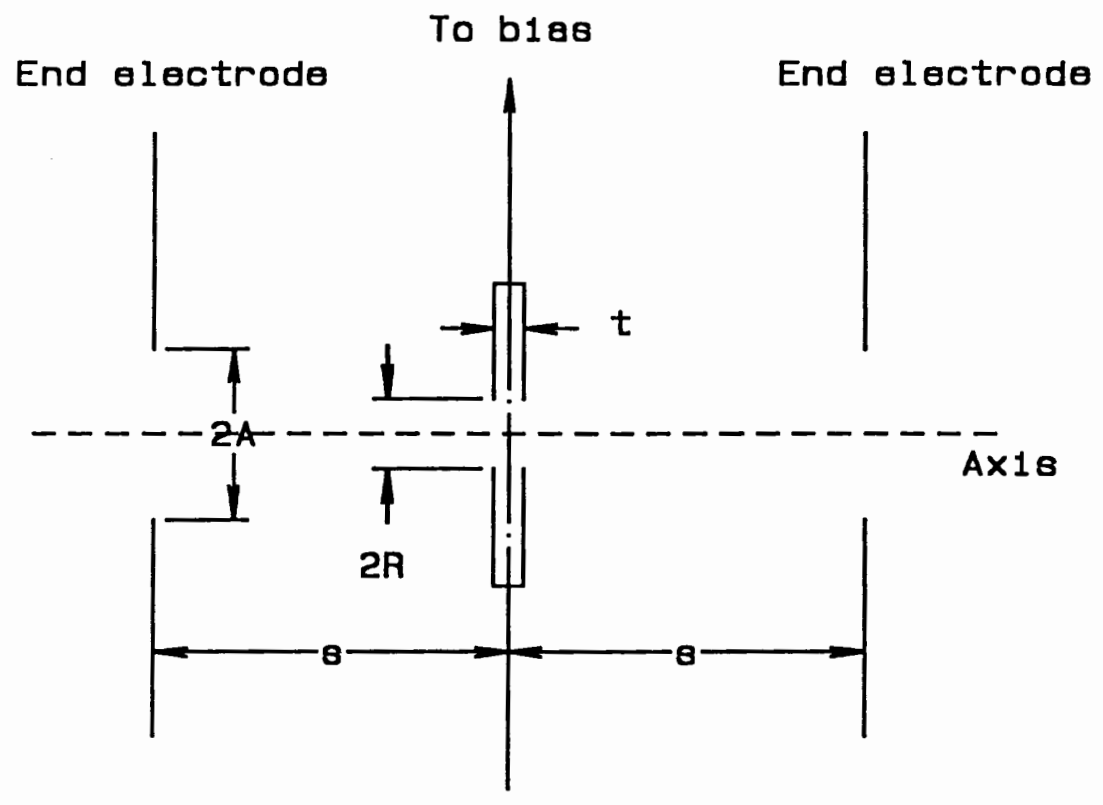

Table of dimensions

\begin{tabular}{|c|c|c|}
\hline Symbol & Value & Value \\
\hline $2 \mathrm{~A}$ & $3.81 \mathrm{~mm}$ & $3.81 \mathrm{~mm}$ \\
\hline $2 \mathrm{R}$ & $1.0 \mathrm{~mm}$ & $0.4 \mathrm{~mm}$ \\
\hline $\mathrm{t}$ & $1.27 \mathrm{~mm}$ & $0.51 \mathrm{~mm}$ \\
\hline $\mathrm{s}$ & $5.84 \mathrm{~mm}$ & $5.84 \mathrm{~mm}$ \\
\hline
\end{tabular}

Figure 11. Essential dimensions of the compound apertures used in the analyzer 


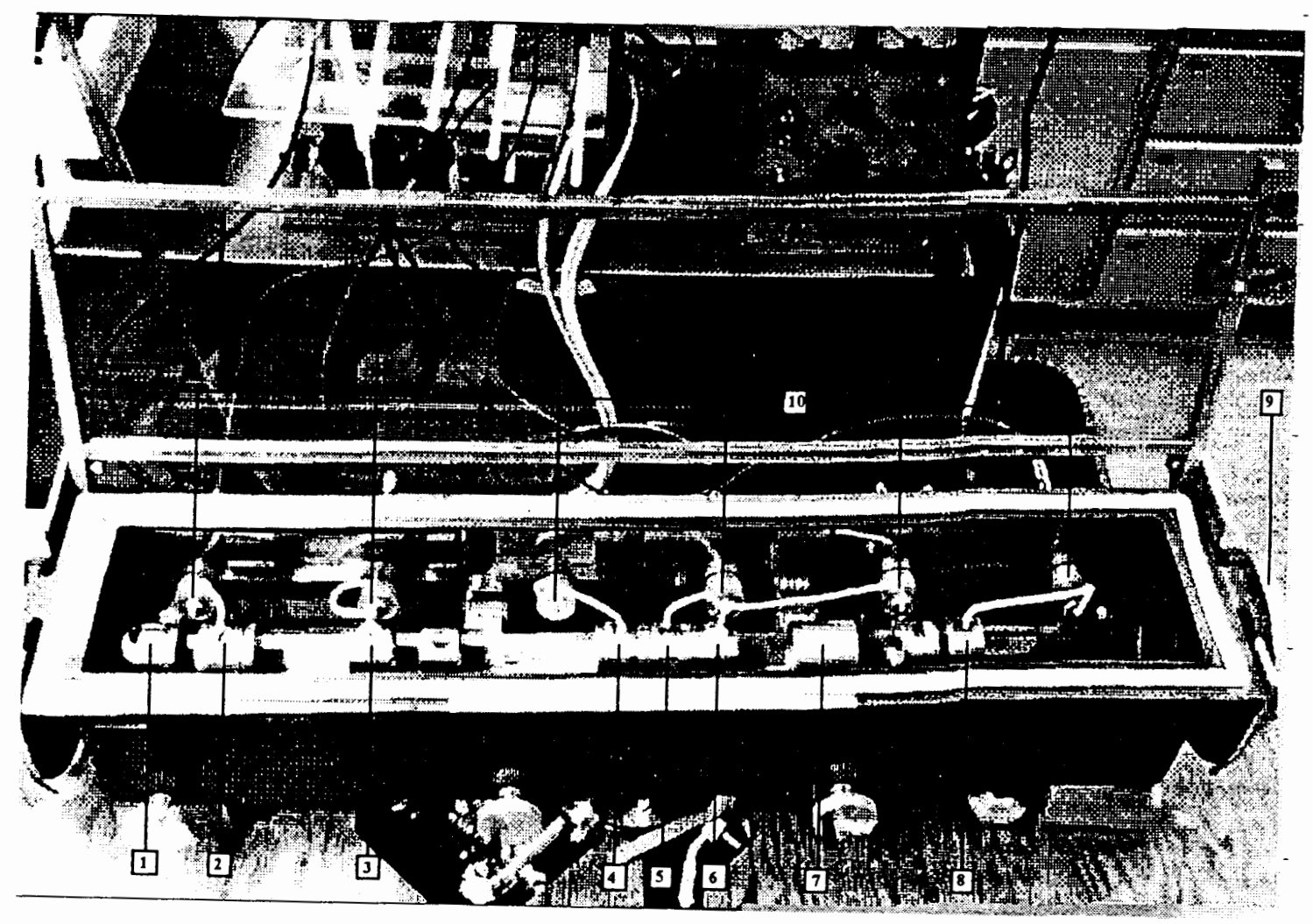

1 -... Electron gun

3 -.-. Field lens

5 --.- Analyzer

7 --.- Shield

9 -..- Screen
2 -..- Condenser lens

4 -.-- Input lens

6 ---- Output lens

8 -.-- Projection lens

10 ---- Feedthroughs

Figure 12. Photograph of experimental system setup. 
the field lens. The output lens (6) projects an image of the retarding electrode to a projection lens (8). The projection lens is used to provide an enlarged image at the final screen or camera. Without enlargement, the image would be inconveniently small for viewing or for measurements. Electrical feedthroughs (10) at the back of the bench supply voltages to the lenses and the retarding electrode. Mechanical controls on the front can be used for positioning components along the V-way. Mu-metal shields line the bench and are also used along the $V$-way to protect against deflections due to external magnetic fields. One V-way shield (7) is shown. A glass top for viewing the experiment can be opened for access to the apparatus.

All of the lenses used in this experiment are electrostatic lenses of the threeelectrode unipotential type. The properties of lenses of this type are given in reference [6]. The focal power is variable depending on the ratio of the lens voltage to the accelerating voltage. The accelerating voltage in the present experiments is $15 \mathrm{kV}$, with the positive terminal at ground potential and the negative terminal isolated from ground at $-15 \mathrm{kV}$. A schematic diagram of the electrical connections is shown in figure 13 . The geometrical dimensions of the electron optical system are given in figure 14 .

\section{ELECTRON-OPTICAL SETTINGS}

The focal power of the electron lenses must be adjusted to meet criteria (a) and (b) of Chapter II, namely that the ray pencil axes be normal to the retarding electrode and that the rays forming the individual pencils focus at the retarding electrode. The procedure for arriving at the appropriate settings is described for the case of the mesh 


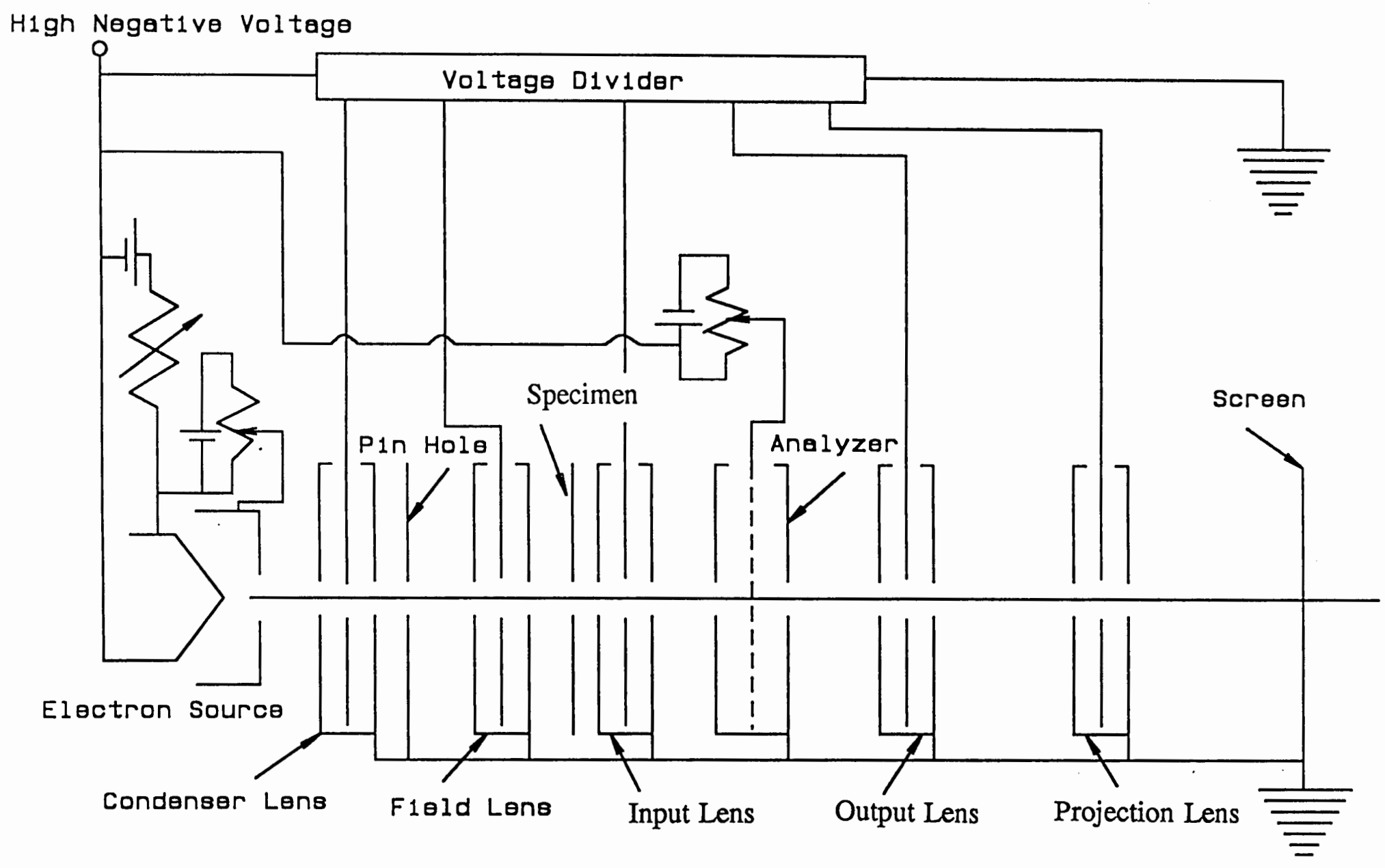

Figure 13. Schematic diagram of the experimental system. 


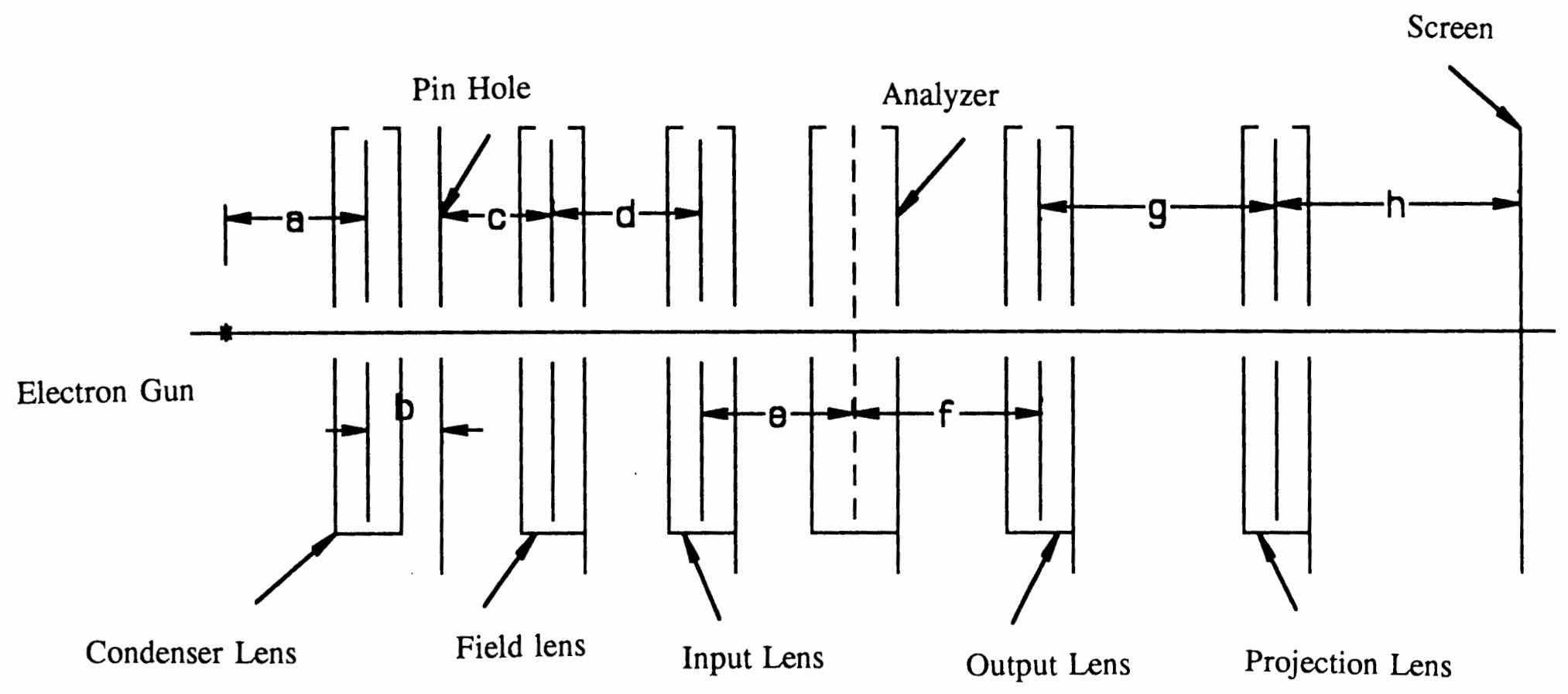

\begin{tabular}{|l|c|c|c|c|c|c|c|c|}
\hline Symbol & $a$ & $b$ & $c$ & $d$ & $\theta$ & $f$ & $g$ & $h$ \\
\hline Value mm & 60.96 & 24.13 & 91.44 & 195.58 & 45.72 & 40.64 & 203.20 & 241.30 \\
\hline
\end{tabular}

Figure 14. Essential dimensions of experimental system. 
electrode. The first step is to adjust the output lens to focus on the retarding mesh, and to adjust the projection lens to provide a convenient magnification. For this purpose the mesh bias is adjusted to permit a portion of the beam to be transmitted. In case the analyzer is to be used as an imaging filter, the input lens is then adjusted to form an image of a specimen at the retarding mesh. In the present experiments the specimen used for this adjustment was a very fine (1000/inch) auxiliary mesh. The focusing of the image at the retarding mesh is monitored by means of the output optics. The field lens is then adjusted so that the ray pencils from the different points of the specimen are normal to the mesh. This condition was judged to be satisfied when the illumination in the mesh openings was same over the field of view and was extinguished at the same retarding bias. The settings made in this process are appropriate also when only the energy distribution, without image formation, is required. The same procedure is used in the case of the compound apertures.

\section{CALIBRATION OF THE ANALYZER}

The analyzer was calibrated by studying its properties for a beam with a narrow distribution of energies. For the calibration experiments the electron gun was used without a specimen in the beam. The electron beam from the gun has an energy distribution with a full width at half maximum (FWHM) of $\sim \mathrm{kT}$. For our gun, with a thoriated tungsten filament, the FWHM is $\sim 0.2 \mathrm{eV}$. This width was narrow enough to use for calibrating the analyzer. The calibration was done by incrementally reducing the bias from cut-off and recording the corresponding images of the retarding mesh or 
compound apertures. The density of the recorded image was taken to be a measure of the transmitted electron current. The transmitted current itself was not measured directly.

Recording was done on a camera located in the vacuum behind a viewing screen which served also as a shutter. The bias voltage ranged from cut-off to zero bias relative to cathode. The incident beam current and exposure times were held constant for each series. The film was advanced behind a strip-shaped beam-stop for each exposure. All of the exposures for a given series were recorded on a single piece of film, thus ensuring uniform processing during development.

Densitometer readings for a given mesh opening or compound aperture were taken for the series of bias settings and plotted as a function of bias voltage. The effective density in the image of an opening was obtained by masking off the surrounding pattern and measuring the transmission of light through the image. The effective density is related to the current of electrons transmitted through the opening.

\section{ENERGY-DISTRIBUTION MEASUREMENT}

After the calibration experiments, the analyzer was used to study a beam having a broadened energy distribution. The broadened distribution was obtained by placing a transmission type specimen in the path of the electron beam. The specimen consisted of a thin evaporated gold layer on a collodion film supported on a $200 /$ inch specimen grid. The energy broadening was due to inelastic scattering in the specimen. The specimen was placed downstream from the field lens, and the beam transmitted by the energy analyzer was measured by the image-density process described in the previous section. The 1.0 
$\mathrm{mm}$ compound aperture was used as the retarding electrode in this experiment.

\section{IMAGING A FILTERED BEAM}

In a further experiment the feasibility of obtaining a focused image, after filtering by the analyzer, was explored. It turned out that the specimen used for the energybroadening experiment was not suitable for testing the possibility of forming an image because the details in the specimen were beyond our resolution capabilities. Instead it was decided to use the 1000 /inch auxiliary mesh as the specimen, without broadening the distribution, and to explore how close the bias could come to cut-off before the image was lost. (The auxiliary mesh was used earlier in setting up the electron optical parameters.) The observations of the image would provide information on how the effects of the converging fields near the apertures in the retarding electrode might limit the resolution. For this experiment also, the electrode with $1.0 \mathrm{~mm}$ compound aperture was used. 


\section{CHAPTER IV}

\section{EXPERIMENTAL RESULTS AND DISCUSSION}

The experimental curves shown in figures 15 to 17 are the calibration curves for the three different retarding electrodes. Each figure is a plot of effective image density as a function of bias voltage from cut-off to zero bias relative to cathode. Figure 15 is the calibration curve for the $600 /$ inch hexagonal mesh. Cut-off is at a bias of -27.7 volts. This result is very close to the theoretical value of -27.9 volts for the conditions of the experiment (see Appendix A). The density curve rises sharply from cut-off (-27.7 volts) to -24 volts, after which the density remains essentially the same, indicating that for biases less negative than - 24 volts there is no appreciable change in transmitted current. The 3.7 volt interval from cut-off to full transmission (rise interval) is due principally to the potential sag in the aperture, that is, to the variation in potential, or barrier height, over the plane of the aperture. As the bias is made less negative the area of the aperture through which electrons are transmitted increases. The local converging fields around the apertures tend to funnel the electrons toward the aperture centers, so the transmitted current saturates well before the entire aperture becomes transmitting. The energy distribution in the beam from the electron gun, with a FWHM $\sim 0.2 \mathrm{eV}$, does not contribute appreciably to the width of the rise interval.

Figure 16 is the calibration curve for the $0.4 \mathrm{~mm}$ diameter compound aperture. 


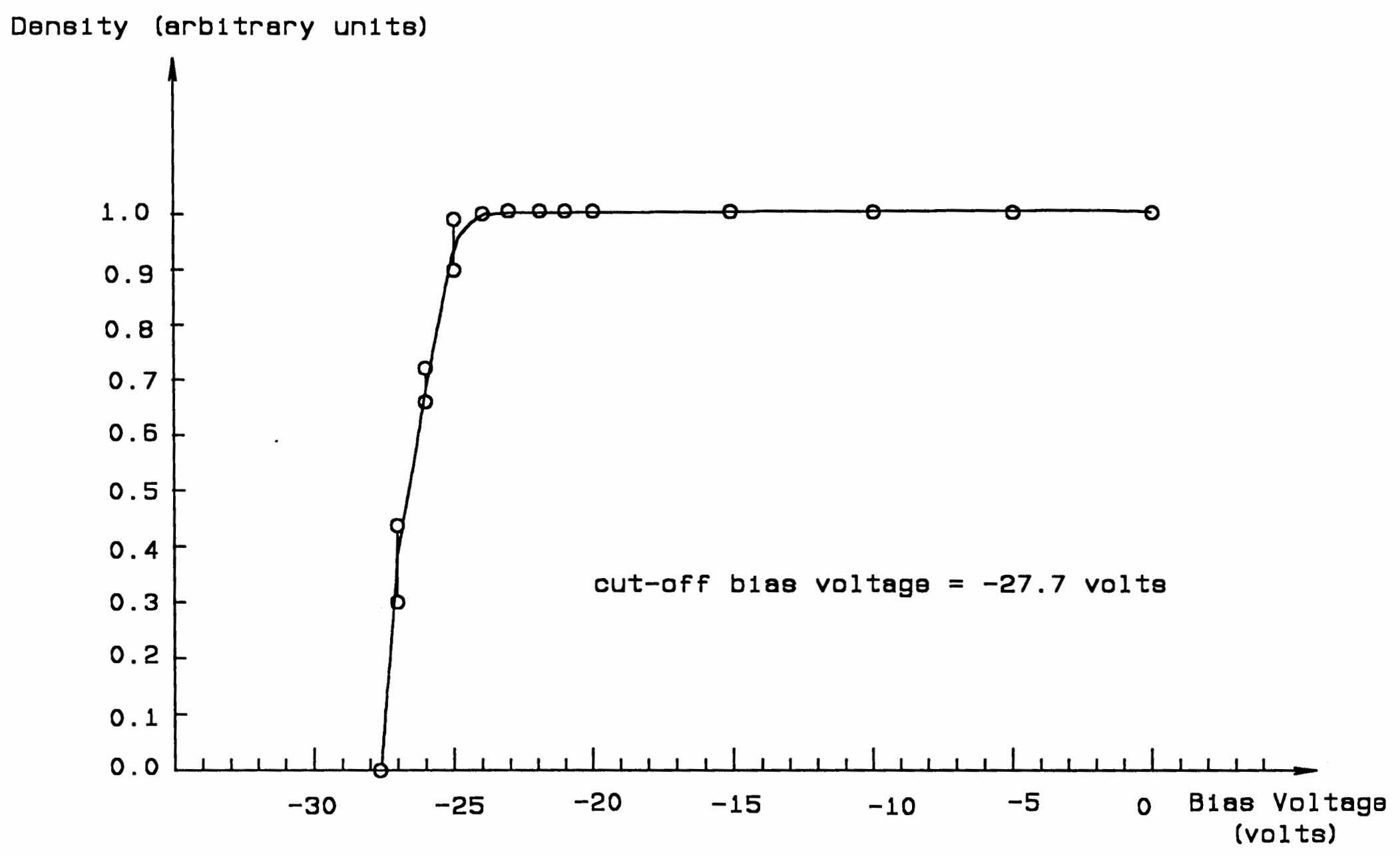

Figure 15. Calibration curve for 1/600" hexagonal mesh. 


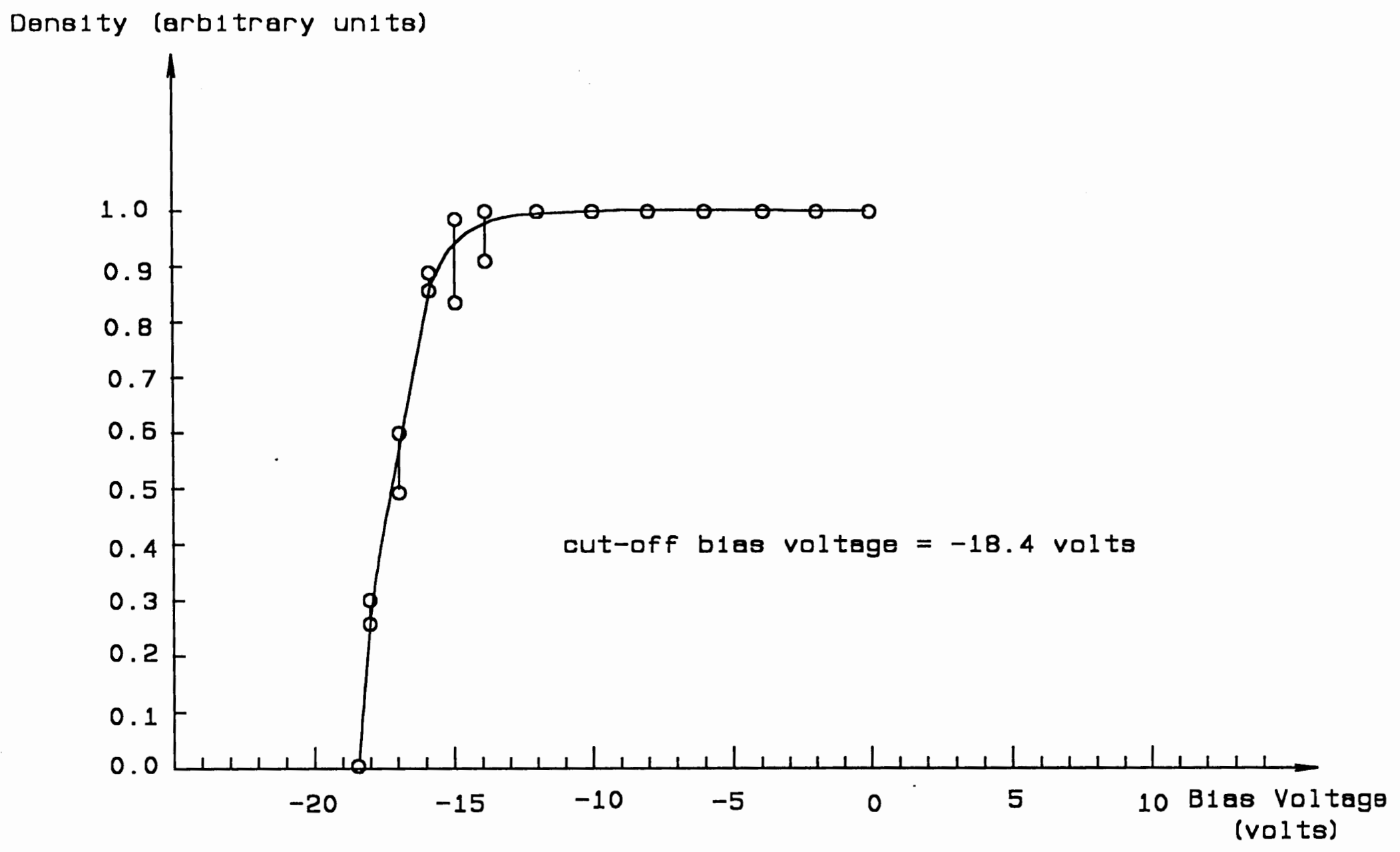

Figure 16. Calibration curve for $0.4 \mathrm{~mm}$ compound aperture 


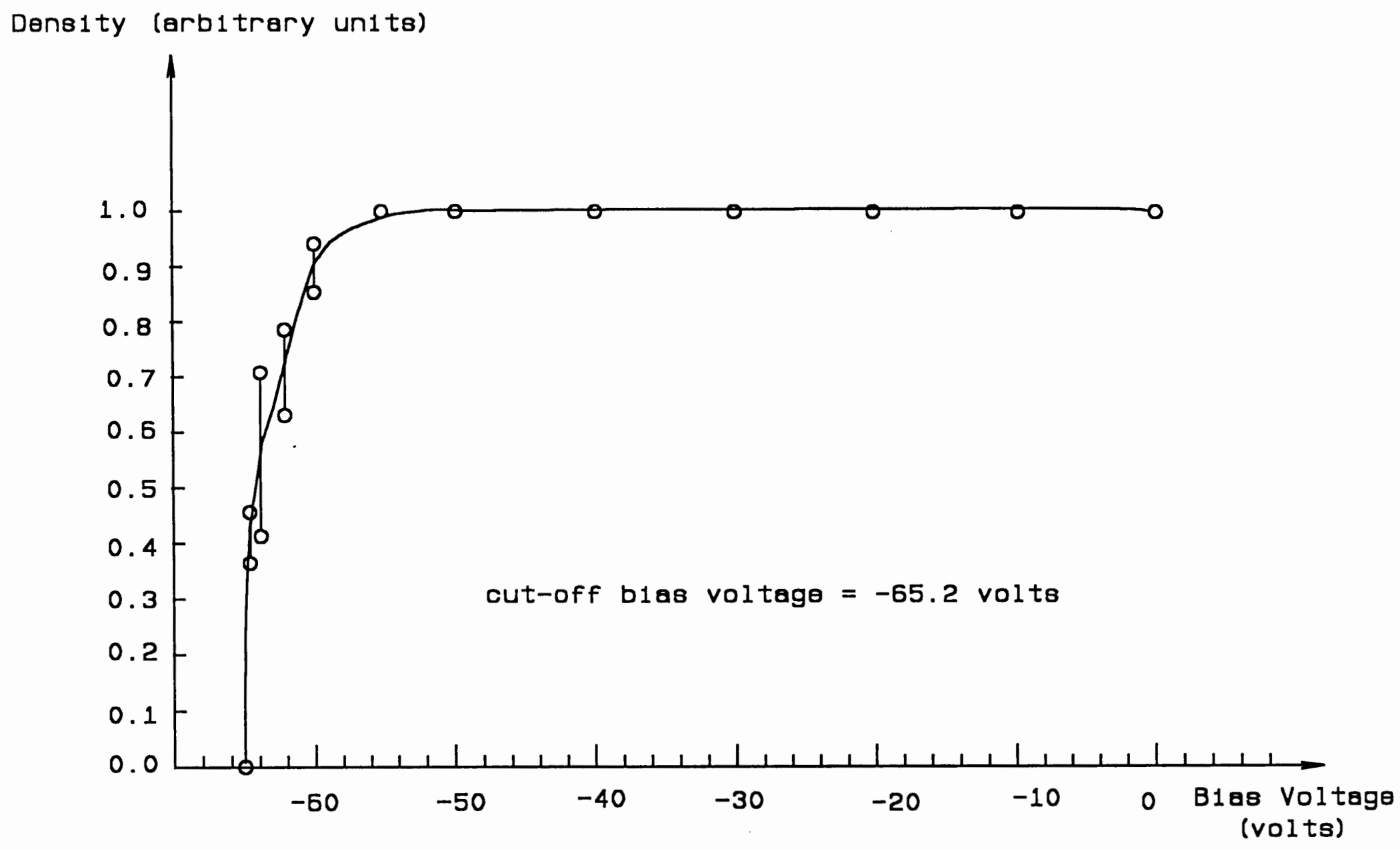

Figure 17. Calibration curve for $1.0 \mathrm{~mm}$ compound aperture. 
The cut-off voltage is - 18.4 volts. The curve rises sharply from cut-off to -14 volts, an interval of 3.6 volts. Figure 17 is the calibration curve for the $1.0 \mathrm{~mm}$ diameter compound aperture. Cut-off is at -65.2 volts, and the curve rises sharply from -65.2 volts to - 60 volts, an interval of 5.2 volts.

The curves in figures 15 to 17 have some similar features, a sharp rise from cutoff, as the bias is made less negative, followed by a leveling off at a density essentially the same as at zero bias.

The curve in figure 18 shows the effect of broadening the energy distribution in the beam. As described in Chapter III, for this experiment a specimen was inserted in the path of the electron beam prior to its incidence on the analyzer. The specimen consisted of an evaporated layer of gold on a thin collodion film supported on a 200/inch grid. This curve was obtained with the $1.0 \mathrm{~mm}$ diameter compound aperture in the analyzer. Comparison with the calibration curve for this aperture (dashed line) shows that significant electron energy losses occur from $0 \mathrm{eV}$ to around $40 \mathrm{eV}$.

In the imaging experiment the 1000 /inch mesh was used as the specimen, and the $1.0 \mathrm{~mm}$ compound aperture was used in the retarding electrode in the analyzer. The portion of the mesh image transmitted by the $1.0 \mathrm{~mm}$ aperture was observed as a function of bias voltage. It was found that the image could be retained up to a negative bias of 1.2 volts from cut-off.

The measurements presented here were made with a $15 \mathrm{kV}$ beam incident on the analyzer. The correspondingly large potential difference required across the analyzer resulted in a large voltage interval from cut-off to full transmission. This was 


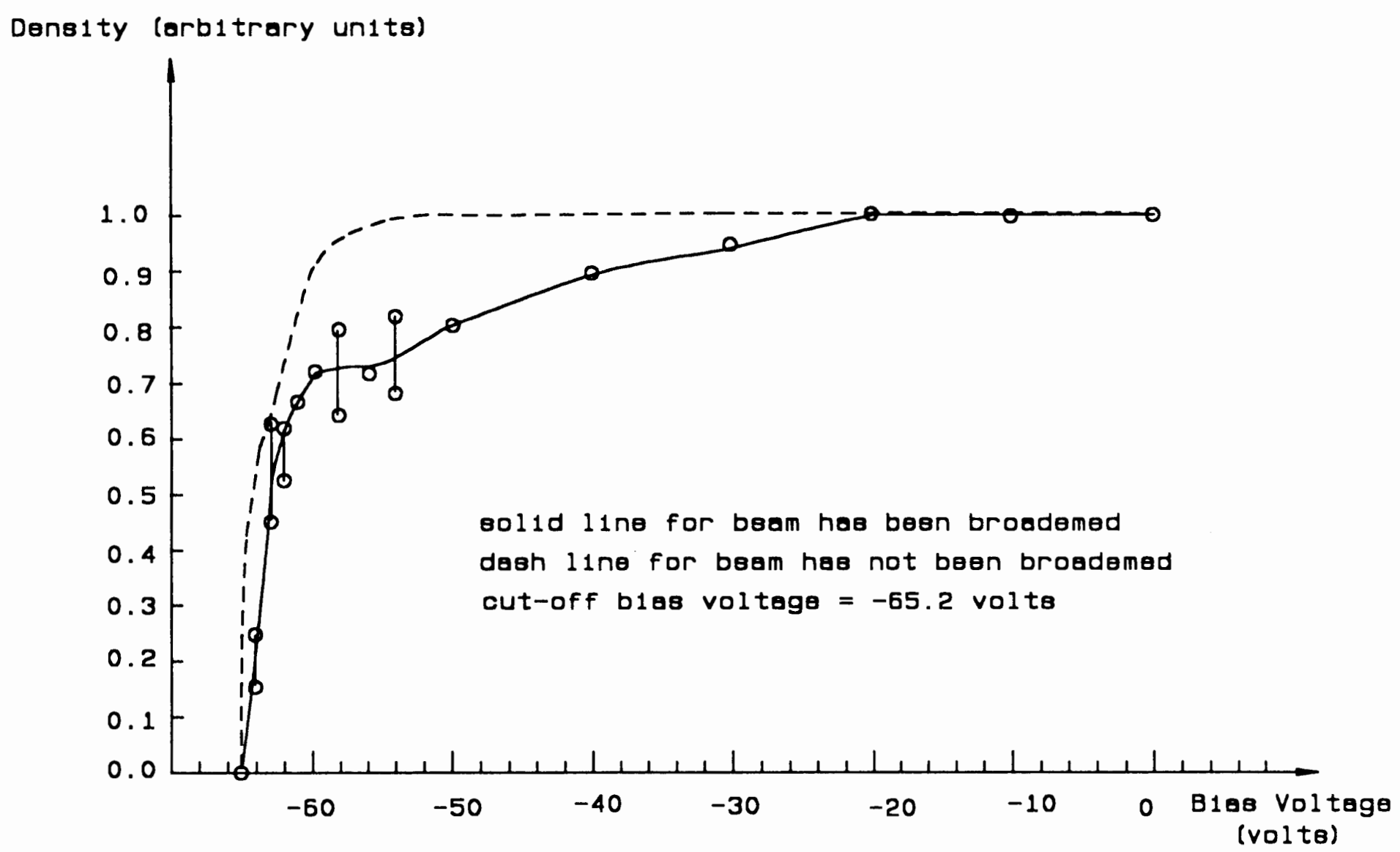

Figure 18. Transmission curve for an energy-broadened beam. The energy distribution of the beam was broadened by passage through a specimen. The $1.0 \mathrm{~mm}$ compound aperture was used in the analyzer. The dashed curve is the calibration curve for the $1.0 \mathrm{~mm}$ aperture.the cut-off bias is 65.2 volts. The curves show that the electrons suffered significant energy losses of from 0 to $40 \mathrm{eV}$ on passage through the specimen, a layer of gold evaporated onto a thin collodion film. 
advantageous for the calibration experiments in that the energy distribution in the electron beam was narrow by comparison with the voltage interval. Conversely, however, the energy resolution of the analyzer is poor under these conditions.

Typically, electrostatic analyzers operate with beams which have been decelerated to low voltages before entering the analyzer and are re-accelerated after leaving the analyzer. In practice the present analyzer also would be used in conjunction with a decelerator-accelerator arrangement. The cut-off bias and the rise interval are proportional to the voltage across the analyzer. For example, for $1.5 \mathrm{kV}$, rather than 15 $\mathrm{kV}$, the cut-off voltage for the $1.0 \mathrm{~mm}$ compound aperture would be -6.52 volts and the rise interval would be 0.52 volts, and for 150 volts these values would be -0.65 volts and 0.05 volts. Furthermore, for a given electron energy, the strength of the focusing field around the retarding aperture would also be reduced proportionately. Thus, both the energy resolution and the prospects for getting a well-resolved filtered image could be greatly improved with low voltage beams.

The practical limit to which the incident beam is decelerated depends in part on a compromise between energy resolution, which improves with voltage lowering, and alignment problems, which become more difficult with voltage lowering (the lower the voltage, the more vulnerable the beam is to spurious deflecting fields). Alignment problems are less difficult to solve with an in-line system than with a system which makes use of beam deflection for energy analysis. This advantage applies especially to an in-line system, such as described in this thesis, which is rotationally symmetric and in which the auxiliary lenses are electrostatic. Furthermore, construction of such a system 
is simpler, and stringent mechanical tolerances are less difficult to meet, than in deflection systems. A disadvantage of a retarding-potential analyzer is that it is high-pass energy filter and therefore to obtain the current within a given interval one must determine the difference in transmission for two bias settings. However, the attractive features of rotationally-symmetric in-line analyzers, along with the results obtained thus far with the retarding-potential method, invite further investigation of the potential of this type of analyzer. 


\section{CHAPTER V}

\section{CONCLUSIONS}

A retarding-potential method of analyzing the energy distribution in an electron beam has been investigated. Three different configurations were used for the retarding electrode: a 600/inch hexagonal mesh, a $0.4 \mathrm{~mm}$ diameter compound aperture, and a 1.0 mm diameter compound aperture. The analyzer was calibrated for each of the retarding electrodes by measuring the transmission of a beam with a narrow distribution of energies as a function the bias voltage on the retarding electrode. The incident beam voltage was $15 \mathrm{kV}$. The calibration curves in each case show the cut-off of transmission at a negative bias voltage with respect to cathode and a sharp rise in transmission over a voltage interval as the bias is made less negative. Following the sharp rise, the transmission levels off at essentially the zero bias value. The values of the cut-off bias for the mesh electrode, the $0.4 \mathrm{~mm}$ electrode, and $1.0 \mathrm{~mm}$ electrode were $-27.7,-18.4$, and -65.2 volts respectively, and the corresponding rise intervals were $3.7,3.6$, and 5.2 volts.

The transmission curve for a beam with a broadened energy distribution was also measured. In this case, the electrode with the $1.0 \mathrm{~mm}$ compound aperture was used. Comparison of this transmission curve with the calibration curve for the $1.0 \mathrm{~mm}$ compound aperture showed that the energy distribution extended over about 40 volts. 
In exploring the feasibility of forming a filtered image of a specimen we found that an image of an object could be observed in the beam transmitted by the $1.0 \mathrm{~mm}$ aperture up to a bias 1.2 volts from cut-off.

As pointed out in Chapter IV, in practice electrostatic analyzers operate on beams which have been decelerated to low energies before entering the analyzers. In the retarding-potential analyzer the cut-off bias, rise interval, and strength of the local aperture fields at the retarding electrode would be reduced in proportion, thus greatly improving the energy resolution and the prospects for a filtered image.

The present analyzer is an in-line rotationally-symmetric system which has certain advantages over deflection systems, with respect to simplicity, mechanical precision, and alignment problems with low energy beams. It is felt that these advantages, along with the present work, warrant further investigations to evaluate the performance of the retarding-potential analyzer coupled with a decelerator-accelerator arrangement enabling the analysis of low energy beams. 


\section{REFERENCES}

[1] K. Siegbahn, in Electron Spectroscopy, ed. D.A. Shirly, North Holland, Amsterdam, 1972, 15.

[2] T.A. Carlson, "Photoelectron and Auger Spectroscopy" Plenum, New York, 1978.

[3] R.F. Egerton, "Electron Energy-Loss Spectroscopy in the Electron Microscope" Plenum, New York, 1986, 30.

[4] J.P. Stewart, Master's Thesis, Portland State University, 1969.

[5] C.J. Davisson and C.J. Calbick, Phys. Rev. 42 (1932) 580.

[6] G.F. Rempfer, J. Appl. Phys. $\underline{57}$ (1985) 2285.

[7] V.K. Zworykin, G.A. Morton, E.G. Ramberg, J. Hillier, and A.W. Vance, "Electron Optics and the Electron Microscope" John Wiley \& Son, 1984. 
APPENDIX A

POTENTIAL DISTRIBUTION DUE TO A CIRCULAR HOLE IN AN

ELECTRODE SEPARATING TWO UNIFORM FIELDS 


\section{APPENDIX A}

\section{POTENTIAL DISTRIBUTION DUE TO A CIRCULAR HOLE IN AN}

\section{ELECTRODE SEPARATING TWO UNIFORM FIELDS}

In figure 19, a circular opening of radius $\mathrm{R}$ is shown in the $\mathrm{xOy}$ plane. The electrode in that plane is assumed to be very thin and at a potential $\mathrm{V}_{\mathrm{o}}$. This electrode separates two electric fields. At large distances from the left and the right of the center of the opening, the fields are assumed to be equal to $E_{1}$ and $E_{2}$, respectively.

According to reference [7], the potential is:

$$
\phi(r, z)=-\frac{1}{2}\left(E_{1}+E_{2}\right) z+\frac{1}{\pi}\left(E_{1}-E_{2}\right)|z|\left(\arctan u+\frac{1}{u}\right)+V_{o}
$$

where

$$
u^{2}=\frac{r^{2}+z^{2}-R^{2}+\left[\left(r^{2}+z^{2}-R^{2}\right)^{2}+4 R^{2} z^{2}\right]^{\frac{1}{2}}}{2 R^{2}}
$$

On the axis, $u=|z| / R$.

and

$$
\phi(0, z)=-\frac{1}{2}\left(E_{1}+E_{2}\right) z+\frac{1}{\pi}\left(E_{1}-E_{2}\right)|z|\left(\arctan \frac{|z|}{R}+\frac{R}{|z|}\right)+V_{0}
$$

At the center of the aperture 


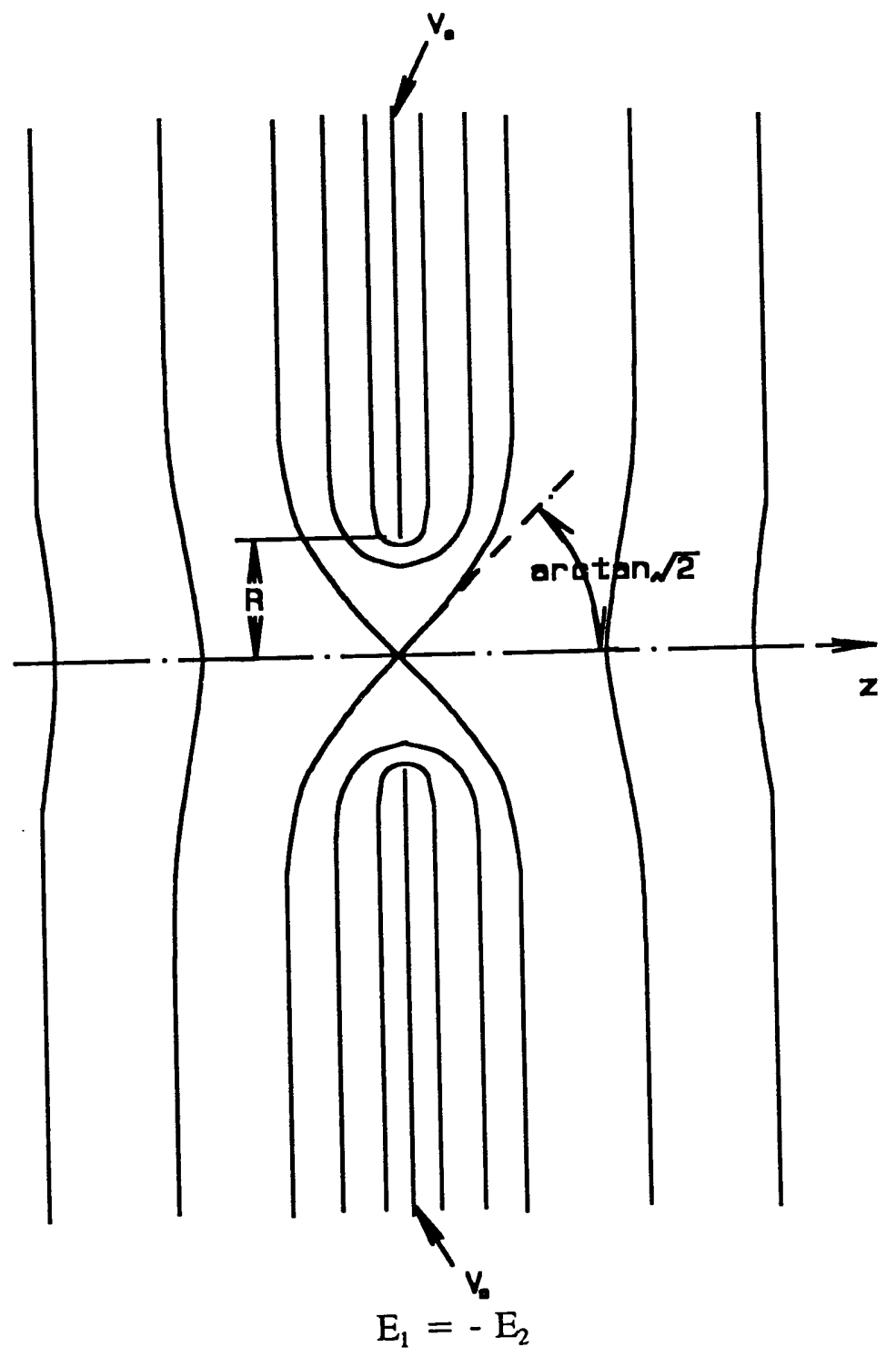

Figure 19. Potential distribution about a circular aperture. 


$$
\phi(0,0)=\frac{R}{\pi}\left(E_{1}-E_{2}\right)+V_{o}
$$

In this experiment, the aperture electrode is between two grounded electrodes spaced a distance $s>R$, so $E_{1}=-E_{2}=-V_{\mathrm{o}} / s$.

where $V_{0}=V_{\text {cathode }}+V_{\text {Bias }}$ is the potential of the aperture electrode.

If the emission kinetic energy of the electrons is negligible, the electron beam is cut-off when $\phi(0,0)-\mathrm{V}_{\text {cathode }}=0$.

For this condition: $\mathrm{V}_{\text {bias }}=2 \mathrm{RV}_{\mathrm{o}} / \pi \mathrm{s}=\mathrm{V}_{\text {cut-off }}$.

For $1 / 600 "$ hexagonal mesh (the transmission rate is $t=0.65$ ), the openings are roughly circular with a diameter $2 \mathrm{R}=\mathrm{t}^{1 / 2} 1 / 600 \mathrm{in} .=0.034 \mathrm{~mm}$,

$$
\mathrm{s}=0.23 \text { in. }=5.84 \mathrm{~mm}, \quad \mathrm{~V}_{\text {cathode }}=15,000 \text { volt. }
$$

The calculated value of $V_{\text {bias }}$ at cut-off is

$$
\mathrm{V}_{\text {cut-off }}=27.9 \text { volt. }
$$


APPENDIX B

THE DAVISSON-CALBICK FORMULA FOR THE FOCAL

LENGTH OF AN APERTURE LENS 


\section{APPENDIX B \\ THE DAVISSON-CALBICK FORMULA FOR THE FOCAL}

\section{LENGTH OF AN APERTURE LENS}

A simple derivation is given here for the focal length of a circular aperture in an electrode with an axial electric field on one side of the electrode. See figure 20.

$$
\begin{aligned}
& 1 / 2 m v^{2}=e V_{B} \\
& \text { ELECTRON BEAM }
\end{aligned}
$$

ELECTRODE AT POTENTIAL $V_{A}$ CIRCULAR APERTURE

$E_{Z_{2}}=\left(-\frac{\partial V}{\partial Z}\right)_{2}=0$

SIDE 2

Figure 20. Circular aperture in an electrode with an axial electric field on one side of the electrode.

Except near the aperture, the field is uniform on side 1, and there is no field on side 2. The incident beam is parallel to the axis. We assume that the potential in the 
aperture does not differ much from the potential $V_{A}$ of the electrode and that the variation is small compared with the beam voltage $V_{B}=m v^{2} / 2$ e of the electrons passing through the aperture. Consequently, as an electron passes through the aperture its distance from the axis does not change appreciably, the angle of deflection is small, and the electron's axial velocity component $\dot{z}$ can be approximated by the total velocity $v$. Also, the beam voltage and axial electric field at the aperture can be calculated as if there were no hole in the electrode.

Consider the case of an aperture in an accelerating electrode. In this case we see that the field around the aperture has a diverging action, as shown in figure 21 . The electron is incident at radius $r$, and is subject to a radial force $(-e) E_{r}=e(\partial V / \partial r)$, where $\mathrm{E}_{\mathrm{r}}$ is the radial component of the electric field. This force produces a radial acceleration $\ddot{r}=(-e / m) E_{r}$. The radial velocity $\dot{r}$ which the electron acquires in passing through the aperture field is given by

$$
\dot{r}=\int_{z_{1}}^{z_{2}} \ddot{r} d t=\int_{z_{1}}^{z_{2}}(-e / m) E_{r} d t=\int_{z_{1}}^{z_{2}}(-e / m) E_{r} d z / \dot{z}=\frac{(-e / m)}{V} \int_{z_{1}}^{z_{2}} E_{r} d z
$$

where, in the last step, $\dot{z}$ has been replaced by $v$, and the constants have been pulled out of the integral. The integration from $z_{1}$ to $z_{2}$ extends out to a distance where the radial field becomes negligible. This integral will be evaluated later. The deflection angle $\alpha$ is given by

$$
\tan \alpha=\dot{\mathrm{r}} / \dot{\mathrm{z}}=\mathrm{r} /(-\mathrm{f}) \text {. }
$$

from which

$$
\mathrm{f}=-(\dot{\mathrm{z}} / \dot{\mathrm{r}}) \mathrm{r}=-(\mathrm{v} / \dot{\mathrm{r}}) \mathrm{r}
$$




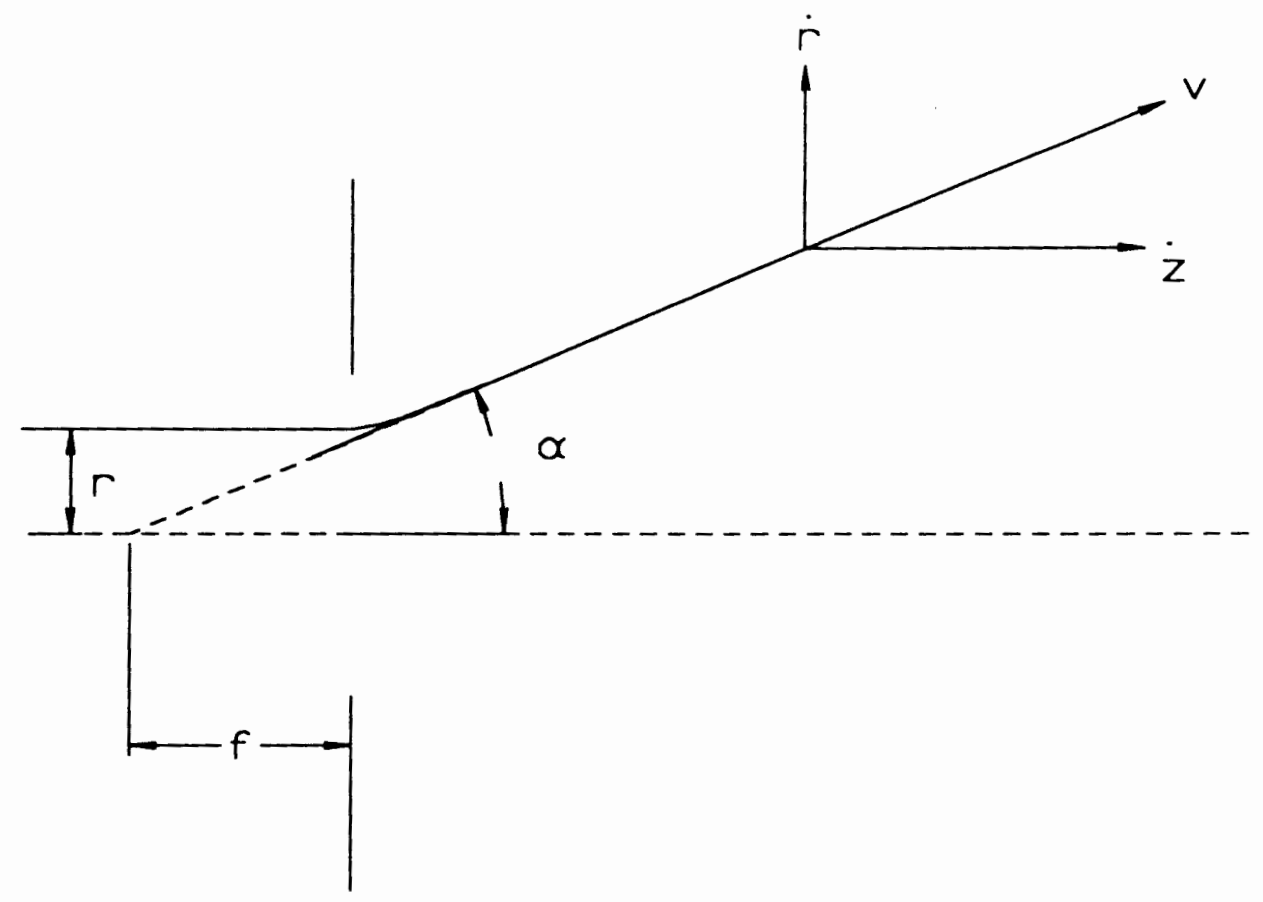

Figure 21. Diverging action around the aperture.

On substituting for $\dot{r}$ from (1),

$$
f=\frac{\left(m v^{2}\right)}{e}\left(r / \int_{z_{1}}^{z_{2}} E_{r} d z\right)=\left(2 V_{B}\right)\left(r / \int_{z_{1}}^{z_{2}} E_{r} d z\right)
$$

where $\mathrm{mv}^{2} / \mathrm{e}$ has been replaced by $2 \mathrm{~V}_{\mathrm{B}}$.

The integral, $\int_{z_{1}}^{z_{2}} E_{r} d z$ can be evaluated by making use of Gauss's law applied to a cylindrical volume of radius $r$ extending over the length of the diverging field from $z_{1}$ to $z_{2}$, as illustrated in figure 22 . Since this volume is charge-free the number of electrons in the volume at any one time is too few to affect the field) the electric flux 
entering the volume, $2 \pi \mathrm{r} \int_{z_{1}}^{z_{2}} E_{r} d z$ must be equal to the flux leaving the volume, $\left(\mathrm{E}_{\mathrm{z} 1}\right)\left(\pi \mathrm{r}^{2}\right)$. Hence,

$$
\begin{gathered}
\int_{z_{1}}^{z_{2}} E_{r} d z=(r / 2) E_{z l} \\
\mathrm{f}=4 \mathrm{~V}_{\mathrm{B}} / \mathrm{E}_{\mathrm{z} 1}=4 \mathrm{~V}_{\mathrm{B}} /(-\partial \mathrm{V} / \partial \mathrm{z})_{1} .
\end{gathered}
$$

If there were a field on side 2 also, the expression for $f$ would be

$$
\mathrm{f}=4 \mathrm{~V}_{\mathrm{B}} /\left\{(\partial \mathrm{V} / \partial \mathrm{z})_{2}-(\partial \mathrm{V} / \partial \mathrm{z})_{1}\right\}
$$
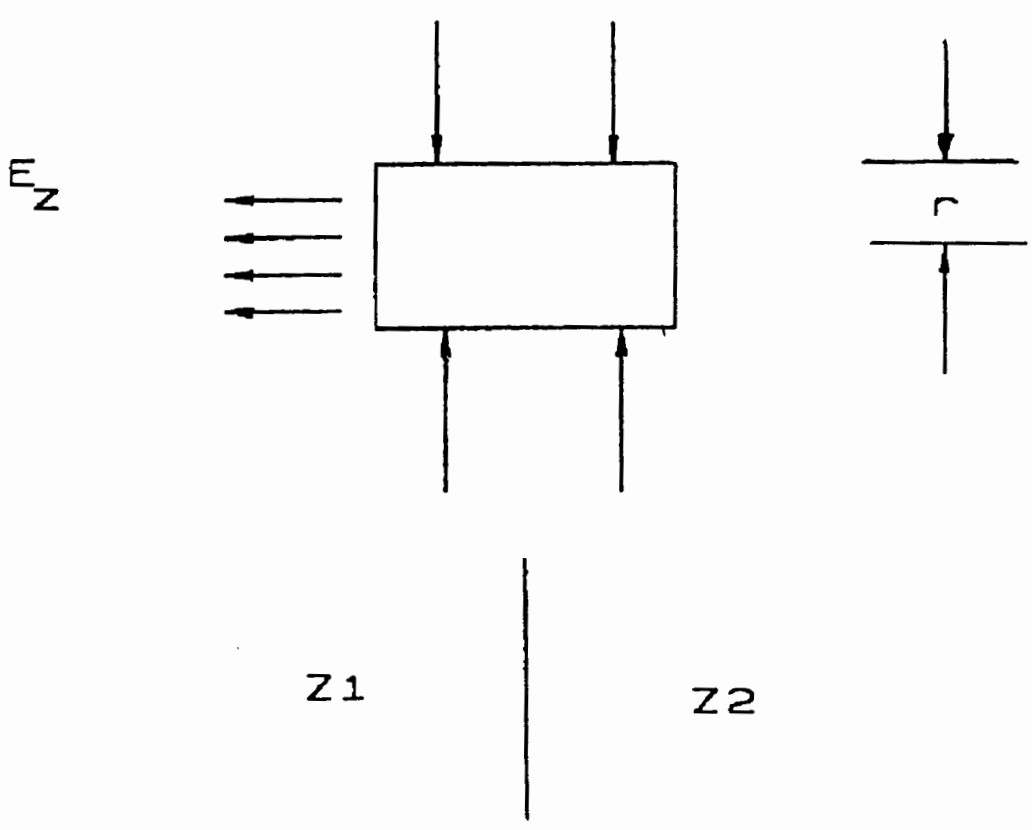

Figure 22. Cylindrical volume diagram for calculating the integral. 